\title{
Comparison of Randomization-Test Procedures \\ for Single-Case Multiple-Baseline Designs
}

\author{
Joel R. Levin, University of Arizona \\ John M. Ferron, University of South Florida \\ Boris S. Gafurov, George Mason University
}

\begin{abstract}
Author Note
The first two authors contributed equally to this study. Correspondence concerning the article should be addressed to Joel R. Levin at jrlevin@u.arizona.edu.
\end{abstract}




\begin{abstract}
In four simulation investigations we examined the Type I error control and statistical power of several different randomization-test procedures for analyzing the data from single-case multiplebaseline intervention studies. Two of the procedures (Wampold-Worsham and Revusky) are associated with single fixed intervention start points and three are associated with randomly determined intervention start points. Of the latter three, one (Koehler-Levin) is an existing procedure that has been previously examined and the other two (modified Revusky and restricted Marascuilo-Busk) are modifications and extensions of existing procedures. All five procedures were found to maintain their Type I error probabilities at acceptable levels. In most of the conditions investigated here, two of the random start-point procedures (Koehler-Levin and restricted Marascuilo-Busk) outperformed the others with respect to detecting immediate abrupt intervention effects; and for all procedures, power was drastically reduced for other types of effects, with the fixed start-point Wampold-Worsham procedure generally faring best. In multiple-baseline designs where it is not feasible or convenient to provide the same or similar series lengths for all cases, the nearly 50-year-old original Revusky procedure should be abandoned in favor of adopting the modified Revusky procedure as a reasonable alternative to the restricted Marascuilo-Busk procedure.
\end{abstract}




\section{Comparison of Randomization-Test Procedures \\ for Single-Case Multiple-Baseline Designs}

The multiple-baseline design is one of the most popular investigative formats adopted by single-case intervention researchers (see, for example, Horner \& Odom, 2014). In addition, because of the design's systematic stagger of the intervention across the replicate "cases" (consisting of individual participants, small groups, schools, or other aggregates), settings/situations, or outcome measures, the multiple-baseline design is generally regarded as the most internally valid [i.e., experimentally sound, scientifically "credible" (Levin, 1994)] design in the single-case interventionist's methodological toolbox (e.g., Kratochwill et al., 2013; Kratochwill \& Levin, 2010). The "most internally valid" claim applies most forcefully to the "across independent cases" variation of the design (Levin, 1992).

As a typical multiple-baseline illustration, consider the design in Figure 1, which consists of 20 observational time periods/sessions, four students, and a systematically staggered instructional intervention (e.g., a novel mathematical problem-solving strategy) with associated outcome measures (percent correct on representative problem-solving tests). In the hypothetical study, all students are observed and tested for a minimum of five sessions in a baseline/control (A) phase/condition (e.g., uninstructed problem solving). Then, in Session 6, one (ideally, randomly selected) student (Student 1 ) begins the instructional intervention (B) phase followed by a problem-solving test, continuing through the $20^{\text {th }}$ session, while Students 2,3 , and 4 remain in the $\mathrm{A}$ baseline condition. B-phase instruction and testing begins for another (randomly selected) student (Student 2) in Session 9, continuing through the $20^{\text {th }}$ session, while Students 3 and 4 remain in the baseline condition. Finally, the last two (randomly ordered) students (Students 3 and 4) receive their B-phase instruction in a similar fashion beginning in Sessions 12 and 15 , respectively. 
The logic of the systematically staggered multiple-baseline design is crystal clear with respect to its outcome believability, in general, and the production of differential/selective intervention effects (Levin, 1992), in particular. As applied to the present example, with a presumed immediately effective instructional intervention, between Sessions 6 and 8, inclusive, Student 1 should exhibit an increase in problem-solving performance that exceeds whatever increase might be expected of Students 2-4 on the basis of Campbell and Stanley's (1966) "maturation" effects, in the form of adaptation, continued practice, etc. Similarly, between Sessions 9 and 11, Student 2 should exhibit a larger performance increase in comparison to Students 3 and 4 . The same goes for Student 3, relative to Student 4, between Sessions 12 and 14; and finally, a performance increase should occur for Student 4 beginning with Session 15.

The plausible attribution of improved problem-solving performance to the problemsolving intervention per se is tied directly to the staggered-intervention component and selective-improvement outcomes of the multiple-baseline design. Traditionally, visual/graphical analysis procedures have been applied to consolidate that attribution (see, for example, Kratochwill, Levin, Horner, \& Swoboda, 2014; Parker, Vannest, \& Davis, 2014). Recommended visual-analysis procedures involve both a "within-case," across time-periods examination (namely, a "horizontal" pre- and post-intervention comparison when "time" comprises the X axis for each case) and a "between-case," across-participants examination of the anticipated sequentially produced intervention effects (namely, a "vertical" comparison)—see Horner \& Odom (2014). Within-case comparisons consist of verifying that each case's change in outcome mean (or "level" in single-case research terminology), slope ("trend"), or variance ("variability") occurs either coincident with or shortly following the point of intervention (Sessions 6, 9, 12, and 15 for the four respective cases of the present example), whereas between-case comparisons similarly focus on these selective "point of intervention" changes while, in contrast, during the same period the performances of the nonintervened cases remain at a baseline level. 
Over the past five decades, various statistical-analysis procedures have been proposed as either alternatives or supplements to visual analyses of multiple-baseline outcome data. Currently viable statistical procedures include principally those derived from regression and multilevel modeling approaches, as well as those couched within the framework of nonparametric randomization tests (see, for example, Kratochwill \& Levin, 2014). The present set of Monte Carlo simulation investigations focuses on the latter (randomization-test) category of statistical procedures, including an examination of some "old (1967 vintage) wine in new bottles." The specific purpose of the present study is to compare the statistical properties (namely, Type I error control and power) of a number of randomization tests that have been proposed over the past five decades for analyzing multiple-baseline data. This in turn will provide readers of Developmental Neurorehabilitation and other single-case intervention researchers with an empirical basis for selecting one multiple-baseline randomization test over another, depending on their situational contexts and practical constraints.

A recent overview of single-case randomization tests, including their rationale and logic, is provided by Ferron and Levin (2014) and by Levin, Ferron, and Gafurov (2014)—see also Edgington and Onghena (2007). For didactic purposes, last to be discussed here is the earliest (between-case) multiple-baseline randomization-test procedure (Revusky, 1967), along with our proposed modification and extension of it. Prior to discussing those two procedures, we will present four within-case randomization tests-specifically, those of Wampold and Worsham (1986), Marascuilo and Busk (1988), Koehler and Levin (1998), as well as a newly developed "restricted" Marascuilo-Busk procedure.

\section{Nonparametric Randomization Test Procedures for Multiple-Baseline Designs}

All of the test procedures to be introduced here incorporate a comparison between the mean (or some other summary measure) of the baseline-phase observations $(A)$ and that of the intervention-phase observations (B). Specifically, for each procedure a set of test-specific A-B 
differences is constructed to form a randomization distribution. In the following section, we make the various procedures concrete by discussing each of them in the context of a hypothetical, computationally transparent, multiple-baseline example described by Koehler and Levin (1998, pp. 209-210). The example includes three classrooms that receive an instructional intervention that is sequentially introduced to the three classrooms, with varying numbers of baseline (or perhaps in the present context, pretest) and intervention outcome observations that produce a total of 10 outcome observations per classroom. To keep our example numerically simple, let us assume that the 10 outcome observations from each classroom consist of the modal student's performance scores. Furthermore, in our example we will assume that for all of the to-bediscussed procedures: (1) the intervention has been posited to improve average classroom performance; (2) the three classrooms have been randomly assigned to the design's three staggered positions (i.e., rows, and often referred to as "tiers"-see, for example, Barton \& Reichow, 2012); and (3) although the various procedures differ in terms of how the actual intervention start points in the design are determined for the three classrooms, those start points turn out to be just prior to Observation 3 for one of the classrooms, Observation 6 for another classroom, and Observation 8 for the final classroom (see Table 1).

We noted at the outset that the multiple-baseline design per se is capable of encompassing a number of different situations, typically with the "cases" represented by participants, settings, or behaviors (see, for example, Horner \& Odom, 2014). However, random assignment to positions within the design is a critical assumption underlying most of the randomization-test procedures discussed here. As is cautioned in the ExPRT (Exce ${ }^{\circledR}$ Package of Randomization Tests) software's User's Guide (Gafurov \& Levin, 2015, p. 14): "If the 'cases' consist of settings, behaviors, test variations, etc. (rather than independent participants, dyads, groups, etc.), then those cases must also have been randomly assigned to the different stagger positions within the design for the randomization statistical tests...to be valid." It is also worth 
mentioning that in many traditional multiple-baseline designs, the researcher decides when the intervention phase is to commence in a "response-guided" fashion (e.g., on the basis of participants producing a stable level of baseline responding). In contrast, the statistical procedures to be discussed here assume that the intervention start points are either fixed or randomly determined on an a priori basis (for additional discussion, see Ferron \& Levin, 2014, pp. 160-161, as well as Hwang, Levin, \& Johnson's, in press, comments in this journal's special issue on single-case intervention research and statistical analysis).

Apart from our present example's stipulations, and as is discussed in greater detail in the General Discussion section, for certain of the procedures (viz., Marascuilo-Busk, restricted Marascuilo-Busk, Revusky, modified Revusky) the length of each case's series must extend to at least one observation past that case's last potential intervention start point (to be explained shortly). Thus, in the present example, there must be at least 3, 6, and 8 observations for Classrooms 1, 2, and 3, respectively. For other procedures (Wampold-Worsham, KoehlerLevin), the length of each case's series must extend to at least one observation past the last potential intervention start point for the final staggered case in the design (i.e., in the present example, there must be at least 8 observations for all classrooms). To simplify the discussion for our example, the series lengths are the same for all classrooms, namely 10 observations apiece. In addition, there are issues associated with missing outcome observations (i.e., incomplete data) throughout the series, including where such missing observations are permitted to occur. In the present study we assume that all cases have no missing observations. In practice, specific rules for incomplete data must be established and/or methods for estimating missing observations applied (see Levin, Evmenova, and Gafurov, 2014, pp. 192-193 and 211; and the General Discussion). 
"Within-Case" Procedures

Wampold-Worsham procedure. The outcome data for our classroom intervention example are presented in the upper portion of Table 2, where the classrooms are listed in the actual order in which the instructional intervention was introduced to the three classrooms (C1, C2, C3). The focal form of randomization for the Wampold and Worsham (1986) model and its associated statistical test is case randomization (Ferron \& Levin, 2014, p. 159), which depends critically on our previously discussed assumption that the $N$ cases have been randomly assigned to the $N$ staggered positions of the multiple-baseline design. With this assumption: (1) there are $N$ ! equally likely orders (permutations) of intervention administration for the $N$ cases; and (2) the designated outcome measure produced by each of these $N$ ! permutations enters into the randomization distribution for the Wampold-Worsham statistical test. Note that with this test the $N$ cases, along with their outcome data, are permuted while the intervention start points remain fixed, thereby producing $N$ ! possible outcomes for the randomization-test distribution. For the present example there are $3 !=6$ possible assignments of the three classrooms to rows of the multiple-baseline design (i.e., Classroom 1 first, 2 second, and 3 third; Classroom 1, 3, 2; Classroom 2, 1, 3; Classroom 2, 3, 1; Classroom 3, 1, 2; Classroom 3, 2, 1).

Consistent with the previously discussed intervention start points for the three classrooms (Observations 3, 6, and 8, respectively), the calculations presented in the upper portion of Table 2 yield the actual A phase means, the B phase means, and the difference between the two (B-A) for each classroom, as well as the averages across classrooms. It may be seen that the average B-A mean difference is 3.434 points. In the lower portion of Table 2 are presented the comparable calculations based on the classrooms' same outcome data but listed in one of the other equally likely orders that could have occurred (here, Classroom 1, 3, 2). That particular order yields an average B-A mean difference of 2.792 points, which is smaller than the just considered actual average mean difference of 3.434 points. Continuing in this 
fashion (though not displayed here), we calculate the B-A mean differences associated with remaining 4 (of 6 total) possible intervention-administration orders. When that is done, it is seen that all 4 of those differences $(2.550,1.820,2.292$, and 2.204 , respectively, for the previously listed classroom orders $2,1,3 ; 2,3,1 ; 3,1,2$; and 3, 2, 1) are also smaller than the actual average mean difference of 3.434. Under the assumption that the randomization distribution contains 6 equally likely mean differences, the probability that the largest one was produced by the actual order of intervention order (Classroom $1,2,3$ ) is equal to $1 / 6=.167$, which also corresponds to the significance probability ( $p$-value) of a corresponding statistical test assessing that the actual mean difference is larger than what would be expected by chance. Insofar as $p=$ .167 exceeds a conventional Type I error probability $(\alpha)$ such as $.01, .05$, or even .10, one would conclude that the actual outcome here was not statistically significant and instead would regard it as a "chance" finding.

We should mention an issue that applies to all of the multiple-baseline test procedures that will be discussed here, namely, how to deal with randomization-distribution observations that are tied. Suppose that in the just-presented example the actually obtained average mean difference were tied with another outcome as being the largest of the six possible. In that the $p$ value is to represent the probability of obtaining an outcome at least as large as the outcome that was actually obtained, then the other tied value would need to be included in the $p$-value calculation, which would result in a significance probability of $p=2 / 6=.333$. When alternatively thought of in terms of rank-ordering the 6 randomization-distribution outcomes from most extreme to least extreme, the two tied largest values would share the ranks 1 and 2 and both be awarded the "midrank" of 1.5.

Marascuilo-Busk procedure. In contrast to Wampold and Worsham's case-randomization scheme, the Marascuilo and Busk (1988) procedure relies on intervention start-point randomization (Ferron \& Levin, 2014, p. 158), a single-case design-and-analysis innovation 
proposed 40 years ago by Eugene Edgington (1975). As a single-case analog to increasing the internal validity of conventional group designs by randomly assigning interventions to participants, Edgington suggested randomly assigning an intervention start point to the case. Inasmuch as that strategy has much to recommend it, it has become a fundamental internalvalidity enhancer in the single-case interventionist's randomization arsenal (see, for example, Levin, Ferron, \& Gafurov, 2014). Marascuilo and Busk adopted the strategy in extending Edgington's original $N=1$ single-case $\mathrm{AB}$ intervention procedure to $N>1$ situations. With the Edgington procedure, in advance of data collection the researcher specifies a range of intervention start points that are "acceptable" from the standpoints of providing a specific number of baseline observations that: (1) is likely to achieve some degree of pre-intervention outcome stability; and (2) is defensible from both practical and ethical perspectives. An actual intervention start point is then randomly selected from that acceptable (or potential) range and assigned to the case.

For our example and as was noted earlier, suppose that the researcher decides on an a priori basis that each of the three classrooms should provide at least two baseline observations and three intervention observations. In addition, the researcher decides that the intervention should be allowed to start anywhere from Observation 3 and through Observation 8 inclusive, thereby resulting in a potential intervention start-point interval containing $k=6$ observations. Finally, and consistent with the Wampold-Worsham example, suppose that the actual intervention start points were randomly selected to occur just prior to Observations 6, 8, and 3 for Classrooms 1, 2, and 3, respectively. In contrast to the previously discussed WampoldWorsham case-randomization test, however, with the Marascuilo-Busk test the $k_{\mathrm{i}}$ potential intervention start points for each case are systematically varied in combination with those of the other cases, while the cases (along with their accompanying outcome data) remain fixed, thereby producing $k_{1} \times k_{2} \times \ldots \times k_{N}=\prod_{i=1}^{N} k_{i}$ possible outcomes for the randomization test (and 
which simplifies to $k^{N}$ when $k$ is the same for all cases). For the present example based on $k=6$ for each classroom, there are $6^{3}=216$ possible intervention start-point combinations across the three classrooms (e.g., Observations 6, 8, and 3 for the three respective classrooms; Observations 4, 6, and 4; etc.; and ranging from Observations 3, 3, and 3 to Observations 8, 8, and 8).

It is important to mention here that although Marascuilo and Busk (1988) regarded their extension of Edgington's procedure as being applicable to both multiple-case AB and multiplebaseline designs (see specifically the title of their article), they cannot strictly claim that for the latter design. Because with their process of with-replacement sampling from specified intervention start-point intervals across cases, there is the possibility that two or more of the cases will end up with exactly the same intervention-phase start point, which of course, does not satisfy the "staggered" logic and requirement of a multiple-baseline design. We will nonetheless apply the Marascuilo-Busk procedure to the present example and address the requisite "stagger" concern in our return to their procedure in the next section.

Our example's design and actual outcome data are presented in Table 3, where it may be seen that although the three classrooms are not listed in the same order as they were in the upper portion of Table 2, for illustrative purposes the summary data associated with the actual intervention start points in the two tables (the upper portion of Tables 2 and 3) were constructed to be exactly the same. As with the previous example, the B-A mean difference associated with each classroom's actual intervention start point (Observations 6, 8, and 3 for the three respective classrooms) is calculated and then averaged across classrooms to yield a value of 3.434. The process continues, with an average B-A mean difference associated with another possible intervention start-point combination (for example, Observations 4, 6, and 4 for Classrooms 1, 2, and 3, which yields a value of 2.956), and so on through all 216 possible classroom/start-point combinations. For the present example it turns out that the outcome 
associated with the start-point combination that was actually implemented (namely, 6, 8, 3, as depicted in Table 3) is the third largest of the 216 randomization-distribution outcomes and so its $p$-value is equal to $3 / 216=.0139$, which is statistically significant based on a one-tailed $\alpha$ of .05.

Restricted Marascuilo-Busk procedure. As was indicated in the preceding section, the Marascuilo-Busk procedure is not applicable to bona fide multiple-baseline designs because of the possibility of selecting the same intervention-start point for two or more cases-a possibility that increases either as the size of the intervention start-point interval(s) decrease(s) or as the number of cases in the design increases. Koehler and Levin (1998, pp. 209 and 212) mentioned the possibility of sampling the various cases' intervention start points without replacement as a way of eliminating that possibility. Under the requirement that $k \geq N$, doing so would reduce the number of possible randomization-distribution outcomes (based on the original "all $k$ equal" formula) from $k^{N}$ to $k ! /(k-N) !=k \times(k-1) \times \ldots \times(k-N+1)$. This "restricted" intervention start-point sampling adaptation of the Marascuilo-Busk procedure neither was pursued empirically by Koehler and Levin nor to our knowledge has yet been considered in the single-case multiplebaseline data-analysis literature. Consequently, it is examined in depth for the first time in the current simulation study.

For the present example, the original unrestricted Marascuilo-Busk number of $6^{3}=216$ randomization-distribution outcomes would be reduced to a restricted Marascuilo-Busk number of $6 \times 5 \times 4=120$. The actually obtained average B-A mean difference outcome is the same as that presented in Table 3 (namely, 3.434), which still turns out to be the third largest mean difference in the restricted randomization distribution. With the total number of randomizationdistribution outcomes now reduced to 120 , the significance probability is therefore equal to $p=$ $3 / 120=.025$, which again is statistically significant with $\alpha=.05$ (one-tailed). It should also be noted that in Table 3 each classroom has been listed in the order of its randomly assigned 
intervention start point (i.e., $\mathrm{O}_{6}, \mathrm{O}_{8}$, and $\mathrm{O}_{3}$ for Classrooms 1, 2, and 3 respectively). However, it is a simple matter for the researcher to list the classrooms in their temporally correct multiplebaseline order $\left(\mathrm{O}_{3}, \mathrm{O}_{6}, \mathrm{O}_{8}\right)$ when presenting the actual study results.

Koehler-Levin "regulated randomization" procedure. Koehler and Levin's (1998) "regulated randomization" procedure combines the random intervention start-point component of Marascuilo-Busk with the case-randomization component of Wampold-Worsham. In addition, it resolves the previously discussed multiple-baseline "stagger" issue by requiring nonoverlapping intervention start-point specifications for the $N$ cases (as is also true with the Wampold-Worsham procedure), which in turn permits case randomization to be valid and computationally straightforward. Because of the required nonoverlapping intervention start-point intervals between cases, the individual intervals of size $k_{i}$ will generally be narrower than will be the corresponding individual Marascuilo-Busk intervals. As a result of the independent randomization of both intervention start points (Marascuilo-Busk) and cases (WampoldWorsham), the number of possible randomization-distribution outcomes is the product of those for the two preceding procedures: namely, $N ! \prod_{i=1}^{N} k_{i}$ in the general situation, and $N ! \times k^{N}$ when the start-point intervals for all cases are the same width (k).

In Table 4 it may be seen that the researcher has decided that there will be $k=2$ potential staggered intervention start points for each of the three classrooms, with each classroom randomly assigned to both a stagger position and a specific start point within the position: here, just prior to Observation 2 or 3 for Classroom 1 (with $\mathrm{O}_{3}$ randomly selected); just prior to $\mathrm{O}_{5}$ or $\mathrm{O}_{6}$ for Classroom 2 (with $\mathrm{O}_{6}$ randomly selected); and just prior to $\mathrm{O}_{8}$ or $\mathrm{O}_{9}$ for Classroom 3 (with $\mathrm{O}_{8}$ randomly selected). With these actual intervention start points, as before the obtained average B-A mean difference can be seen to be 3.434. Another of the possible Koehler-Levin procedure's average B-A mean differences, calculated as though Classroom 3's intervention start point were just prior to $\mathrm{O}_{2}$, Classroom 1's just prior to $\mathrm{O}_{6}$, and Classroom 2's 
just prior to $\mathrm{O}_{9}$, can be verified to be 2.267 . When examined with respect to all 3 ! $\times 2^{3}=48$ outcomes in the randomization distribution, the actually obtained outcome of 3.434 is found to be the most extreme of all 48 , resulting in a $p$-value of $1 / 48=.021$, which once again is statistically significant with $\alpha=.05$ (one-tailed).

\section{"Between-Case" Procedures}

In all of the multiple-baseline randomization-test procedures presented so far, the cases' Phase B - Phase A mean differences are combined and compared with the same differences assuming that the intervention had been administered either to the cases in different designstagger positions (Wampold-Worsham), at alternative potential intervention start points (Marascuilo-Busk, as well as its restricted version), or both (Koehler-Levin). Because such comparisons are based exclusively on B-A differences of various kinds, we have referred to these as within-case procedures. In contrast, although the procedures to be discussed here (Revusky, as well as its proposed modification and extension) also focus on B-A differences, they do so in stages (or "steps") wherein: (1) at the point of the first case's intervention, that case's difference is compared with the differences of the nonintervened cases; (2) at the point of the second case's intervention, that case's difference is compared with the differences of the remaining nonintervened cases; and so on. Because the basis of the Revusky procedure's logic is primarily a direct comparison of the intervened and nonintervened cases' differences, we refer to it as a between-case procedure.

Revusky procedure. We apply the stepwise approach of the Revusky procedure to our hypothetical classroom intervention example, as is summarized in Table 5. It is clear from reading the procedure's theoretical rationale (Revusky, 1967, p. 322) that both to preserve the design's internal validity and to justify the assumption that all $N$ ! possible outcomes entering into Revusky's randomization distribution are equally likely, cases must be randomly assigned to the various positions within the multiple-baseline design. In addition, and as a reminder of what was 
noted earlier in the present manuscript, the Revusky procedure does not require all cases to complete the same full-length series. Indeed, following each step of the analysis, the justintervened case is excluded from all subsequent comparisons-which means that the case's outcome data from that point on are essentially "wasted" (at least as far as the formal statistical analysis is concerned). Nonetheless, for purposes of consistency with the other randomizationtest procedures discussed so far, we continue to provide the complete set of 10 outcome measures for all three cases that are presented in Table 5. Once again in this example, and as may be seen by the vertical intervention start-point lines in Table 5, we assume that Classroom 1 was randomly assigned the first position in the design (i.e., intervention just prior to $\mathrm{O}_{3}$ ), Classroom 2 to the second position (intervention just prior to $\mathrm{O}_{6}$ ), and Classroom 3 to the third position (intervention just prior to $\mathrm{O}_{8}$ ),

Below the data in Table 5, we see that the Revusky procedure is based on calculating the cases' mean B-A differences at each step, comparing those differences, and rank-ordering them from largest (1) to smallest (3 in Step 1, 2 in Step 2, and not needed in Step 3). It is worth mentioning at this point that Revusky (1967, p. 324) also convincingly argued that in situations where the different cases' baseline $(A)$ performances are at quite different levels, it might make more sense to replace their simple differences $(B-A)$ with relative differences $[(B-A) / A]$, to represent each case's proportional change from the A to B phase. ${ }^{1}$ Although Revusky used standard tables of the Mann-Whitney U statistic (e.g., Siegel, 1956) to conduct the randomization test back in 1967, the actual score calculations are straightforward, as is now illustrated.

With the Revusky procedure, the distribution of summed ranks can range from 3 (1s on all steps) to 6 (3 on Step 1, 2 on Step 2, and 1 on Step 3). Of interest is the rank sum produced by the case that was actually intervened on each step. In the present example, the actual intervention order was Classroom 1 first (rank of 1 on Step 1), Classroom 2 second (rank of 1 
on Step 2), and Classroom 3 third (rank of 1 on Step 3), thereby yielding a rank sum of 3. Because 3 is the lowest total of all $N !=6$ possible rank sums in the randomization-test distribution, it is apparent that the actually intervened cases produced the most extreme B-A mean difference outcome. Despite that, however, with a significance probability of $1 / 6=.167$ the outcome is not statistically significant based on $\alpha=.05$ (one-tailed).

Modified Revusky procedure. In the present study, along with the other multiple-baseline randomization tests discussed so far, we will be investigating the statistical properties (namely, Type I error control and power) of a newly developed modification and extension of the Revusky test: specifically, one that represents a combination of a Revusky between-cases comparison and a Koehler-Levin randomized intervention start-point within-cases comparison. Although the new procedure encompasses the Revusky stepwise approach to calculating B-A mean differences, it relies on a single omnibus randomization test (rather than on Revusky ranks calculated at each step) to assess the statistical significance of the actually obtained B-A mean differences.

Let us now apply our modified procedure to the example in Table 5 with only $k=1$ intervention start point for each of the three cases. Instead of ranking the cases' mean B-A differences at each step (as was done for the original Revusky procedure), we assemble the $N$ ! possible stepwise combinations in a single randomization-test distribution, as follows: As may be seen in Table 5, the three stepwise mean B-A differences associated with the actual outcomes are 2.500 (Step 1), 2.500 (Step 2), and 3.476 (Step 3), for an average of 2.825 . Another possible average mean difference (produced by Step $1=2.500$, Step $2=-0.500$, Step $3=3.476$ ) is 1.825 ; and the four remaining average mean differences may be calculated as $1.714,0.714,1.992$, and 0.992 . In that the actual B-A average mean difference of 2.825 is the largest of the six, the $p$-value of $1 / 6=.167$ for the modified Revusky test coincides with that of the original Revusky test and is again not statistically significant with $\alpha=.05$ (one-tailed). 
The modified Revusky procedure also lends itself to a straightforward extension of the original procedure. Specifically, it affords a randomization test for situations in which a range of potential intervention start points is designated for each case (as with the Koehler-Levin procedure), as opposed to each case having only one predetermined fixed intervention start point (as was just illustrated). As with the Koehler-Levin procedure, this Revusky modification and extension includes a total of $N ! \prod_{i=1}^{N} k_{i}$ possible mean difference outcomes in its randomization-test distribution, which simplifies to $N ! \times k^{N}$ when all cases have the same number of potential intervention start points $(k)$.

That type of situation and its calculations, applied to the present example, are summarized in Table 6 . There it may be seen that each of the three classrooms is associated with two potential intervention start points, one of which is randomly selected as the actual intervention start point for that classroom. This results in a total of $3 ! \times 2^{3}=48$ possible meandifference outcomes in the randomization-test distribution. If, as in the example presented in Table 5, Observations 3, 6, and 8 represent the actual intervention start points for Classrooms 1,2 , and 3 , respectively, then the average $B-A$ mean difference for that specific combination is 2.825, as it was previously. From Table 6 it may be calculated that had the three respective classrooms instead started their interventions with Observations 3, 5, and 8, then the average B-A mean difference would have been $(2.500+1.667+3.476) / 3=2.548$. When these two differences are included with the 46 other possible outcomes in the randomization-test distribution, it is found that our actually obtained outcome of 2.825 is the largest of all 48 , resulting in a significance probability of $1 / 48=.021$, which is statistically significant with $\alpha=.05$ (one-tailed).

\section{Purpose of the Present Study}

As was noted earlier, the focus of the present study is to compare the statistical properties (specifically, Type I error control and power) of the modified Revusky procedure with 
the original Revusky multiple-baseline randomization-test procedure, as well as with the Wampold-Worsham, Koehler-Levin, and the conceptualized-but-not-yet-examined restricted Marascuilo-Busk procedures. A previous study by Ferron and Sentovich (2002) compared the Type I error and power characteristics of the original Marascuilo-Busk, Wampold-Worsham, and Koehler-Levin procedures and found that: (1) all three controlled Type I error rates at acceptable levels; and (2) the Koehler-Levin procedure was slightly (though not dramatically) more powerful than both the Marascuilo-Busk and Wampold-Worsham procedures. At the same time, it should be reiterated that the Marascuilo-Busk is not a true "multiple baseline" procedure because its sampling of intervention start points with replacement allows for the possibility that two or more of the $N$ participants end up with exactly the same intervention start point, a condition that is not compatible with the logic of a multiple-baseline design.

Also, in the Ferron and Sentovich (2002) study, whereas the Marascuilo-Busk procedure was examined under a reasonable range of potential intervention start points for each case, the Koehler-Levin procedure was examined exclusively with the specification of only two potential intervention start points for each case. Thus, there is no indication of how the Koehler-Levin procedure's power would fare with a larger number of potential start points. That issue is addressed in the present investigation.

Finally, in both the Ferron and Sentovich (2002) study and most previous statistical power investigations of single-case randomization tests, the B-phase outcome data have been simulated to represent immediate abrupt changes in the series' levels (means). That is, the Aphase observations are modeled to change immediately following the point of intervention, from a mean effect size of 0 to $d(>0)$, where $d$ is the difference between the case's B and A phase means, divided by an A-phase standard deviation that remains constant throughout the series of B-phase observations (see also Busk \& Serlin, 1992). Other common types of single-case mean level effects include immediate gradual changes (i.e., an increasing level throughout the B 
phase), delayed abrupt changes (i.e., a constant change in level that is evident only two or more observations following the point of intervention), and delayed gradual changes (an increasing level throughout the B phase that begins only two or more observations following the point of intervention). Immediate gradual and delayed abrupt effect-size types are examined in the present study's final set of power simulations (Investigation 4).

\section{Investigation 1: Comparison of Three Procedures With a Fixed Intervention Start Point} Method

Monte Carlo methods were used to examine the Type I error control and power of the Wampold-Worsham, Revusky, and modified Revusky one-tailed randomization tests. We considered multiple-baseline designs with the number of participants ranging from four to six, and the series lengths being 19,22 , or 25 . The choice of these values was motivated by a survey of multiple-baseline studies that found the modal number of participants was four and the median series length was 24 (Ferron, Farmer, \& Owens, 2010). In our four-participant $(N=4)$, 19-observation design, the intervention was staggered with either $s=1$ observation between intervention start points (i.e., start points of $6,7,8$, and 9), $s=2$ observations between intervention start points (i.e., start points of $6,8,10$, and 12), or $s=3$ observations between intervention start points (i.e., start points of $6,9,12$, and 15). For the $N=5$ design, the series length was 22 and the start points were staggered by one (i.e., 6, 7, 8, 9, and 10), two (6, 8, 10, 12 , and 14) or three $(6,9,12,15$, and 18). Finally, for the $N=6$ design the series length was 25 and again the intervention start point for the first participant was 6 and the intervention start point for each successive participant increased by one, or two, or three observations.

Time-series data were generated for each participant using SAS IML (SAS, 2013). A normally distributed error series was created for each participant using the autoregressive moving-average simulation function (ARMASIM), where the autocorrelation in the error series was set either to $\rho=0$ or $\rho=.30$. The choice of autocorrelation values was motivated by our 
wanting to examine values both above and below the average bias-adjusted autocorrelation of .20 that was found in a survey of single-case studies (Shadish \& Sullivan, 2011) and to use values that had been used in previous simulations of multiple-baseline data (e.g., Ferron \& Sentovich, 2002; Ferron \& Ware, 1995). In all simulations the standard deviation of the independent portion of the error series was set to 1.0. The observed data for our simulated studies was created by adding the error series created using the ARMASIM function to an effect-size series, which was coded to have values of 0 for all baseline observations, and values of the earlier defined $d$ for all intervention phase observations; consequently, $d$ indexes the effect of the treatment. The value of $d$ was varied to examine both Type I error control (when $d=$ 0 ) and power ( $d$ s ranging from .5 to 4 in increments of .5). The choice of $d$ values was informed by a survey of single-case intervention studies reported by Parker and Vannest (2009), where the estimated values of $d$ (assuming no autocorrelation for simplicity and expected maximum powers) for the $10^{\text {th }}, 50^{\text {th }}$, and $90^{\text {th }}$ percentiles were $0.46,1.70$, and 3.88 , respectively.

With the number of participants $(N=4,5,6)$ crossed by the size of the staggers $(s=1$, 2, 3 observations), the level of autocorrelation $(\rho=0, .30)$, and the effect size $(d=0$ to 4 , in increments of .5), 162 unique conditions were formed. For each condition, data for 10,000 "studies" were simulated. The data for each simulated study were then analyzed by three randomization tests: the Wampold-Worsham, Revusky, and modified Revusky procedures. For each test, the obtained test statistic was based on the appropriate B-A mean difference, as was described in the previous examples (see also Tables 2, 5, and 6). The Type I error (when $d=0$ ) or power (when $d>0$ ) was estimated for each test for each of the 162 conditions by determining the proportion of the 10,000 simulated studies where the test resulted in a one-tailed $p$-value of .05 or less. 
Results

The results, presented in Figures 2 and 3 (for $N=4$, with autocorrelations of 0 and .30, respectively) and Figures 4 and 5 (for Ns $=5$ and 6 , respective autocorrelations of 0 and .30), are straightforward and therefore easy to describe. First, however, it is apparent from Figures 2 and 3 that the curves associated with the Revusky and modified Revusky tests are identical. Although that identity was not recognized at the outset of our project, we now realize that: (1) for $N=4$, which yields 24 possible outcome permutations, only the single most extreme outcome can be included in an $\alpha=.05$ (one-tailed) rejection region (i.e., 1/24 $=.042$ ); and in that situation: (2) if the Revusky test's most extreme outcome is in that region, then so too will be the modified Revusky test's. As soon as either $N>4$ with one potential intervention point (e.g., Figures 4 and 5) or there are other combinations of $N$ and the number of potential intervention start points that produce more than one outcome in the rejection region, then the Revusky and modified Revusky curves will not always look the same.

Type I error control. As may be seen in Figures 2-5, with an effect size given by $d=0$, for both autocorrelation levels ( $\rho$ s $=0$ and .30$)$ and the three levels of start-point stagger $(1,2$, and 3 observations), the Wampold-Worsham, Revusky, and modified Revusky procedures all produced "acceptable" rejection probabilities (i.e., average Type I error probabilities), namely, empirical as ranging from .026 to .053 . These results in turn indicate that all three procedures provide appropriate Type I error control when used to conduct a multiple-baseline randomization statistical test. Generally speaking, the original Revusky procedure based on $N=5$ and 6 cases was associated with the most conservative (lowest) empirical as.

Power. First, in Figures 2-5, each based on a single fixed intervention start point $(k=1)$ for all cases, it may be seen that the within-case Wampold-Worsham test (the $k=1$ specialsituation manifestation of the Koehler-Levin procedure) is more powerful than the two Revusky between-case tests, the latter two of which are (as per our earlier discussion) identical for $N=4$ 
cases. At the same time, in Figures $4(\rho=0)$ and $5(\rho=.30)$, it is also clear that for $N$ s of 5 and 6 the present modified Revusky test is more powerful than the original Revusky test.

Second, stagger matters. Within each sample size, as the between-case stagger (i.e., between-case separation) increases from one sequentially adjacent intervention start point to each successive case being separated by three observations, all of the tests' powers increase substantially. A straightforward explanation for this is that with more stagger, there are more Bphase observations for each case before the next case's B phase commences and, as a result, the separate cases' B-A phase differences become more distinctive. This is critical for documenting effects in multiple-baseline designs and leads to the observed test statistic being more reliably positioned within the randomization distribution. At the same time, for every level of stagger in Figures 4 and 5, both the Wampold-Worsham and modified Revusky tests based on $N=5$ are seen to be more powerful than the original Revusky test based on $N=6$.

\section{Investigation 2: Comparison of Three Procedures With}

\section{Randomly Selected Intervention Start Points}

Method

In Investigation 1 we documented the statistical power differences among the three multiple-baseline randomization test procedures when a single staggered intervention start point was allocated to each case. In Investigation 2, we examine the Type I error control and power of the Koehler-Levin (an extension of Wampold-Worsham) and modified Revusky randomization tests when an intervention start point, randomly sampled from a staggered set of "acceptable" or "potential" ones, is allocated to each case. To this investigation we added the restricted Marascuilo-Busk procedure (i.e., based on the earlier-discussed sampling-without-replacement modification), for which intervention start-point selection would be "restricted" to ensure no between-case overlap (i.e., between-case intervention start-point staggers of at least one 
observation). Here we consider designs with $N s=4$ and 5, with associated series lengths of 19 and 22 , respectively.

In contrast to Investigation 1, here the potential intervention start points were not fixed in advance, but for the Koehler-Levin and modified Revusky procedures were selected randomly from either $k=2$ or 3 possible start points per case. Thus, for our $N=4$ design with two potential intervention start points per case, the start points for the four cases were selected from $\{6,7\},\{9,10\},\{12,13\}$, and $\{15,16\}$, respectively, which resulted in between-case staggers of between two and four observations. For the three potential start-point variation of the $N=4$ case design, the start points were selected from $\{6,7,8\},\{9,10,11\},\{12,13,14\}$, and $\{15,16,17\}$, resulting in between-case staggers of between one and five observations. For $N=5$ cases, we added the start-point set of $\{18,19\}$ and $\{18,19,20\}$ for the two and three potential start-point variations, respectively. For the restricted Marascuilo-Busk procedure, the potential intervention start-point interval included Observations 6 to 15 inclusive in the $N=4$ design, and from Observations 6 to 18 inclusive in the $N=5$ design, with the actual start point for each successive case randomly selected without replacement.

Time-series data were generated for each case using the same method as in Investigation 1. For all simulations, the autocorrelation was set to .30 and effect sizes were varied from 0 to 4 in increments of .50 . The results for Investigation 1 documented that with an autocorrelation of $\rho=0$, Type I error is well controlled for all sample sizes (see Figures 2 and 4). In addition, although the powers were uniformly higher for $\rho=0$ than for $\rho=.30$ (Figures 3 and 5), it is evident that the respective test-procedure profiles are virtually the same for both levels of autocorrelation (compare Figure 2 with Figure 3 and Figure 4 with Figure 5). For those reasons (and as has been confirmed by additional simulations), in this and in the remaining investigations we present results only for the more realistic single-case research situations, namely, for $\rho=.30$. 
For each design variation, data for 10,000 "studies" were simulated and then analyzed with the corresponding randomization test(s). More specifically, for designs with independently randomly selected intervention start points for each case, the Koehler-Levin and modified Revusky tests were conducted; whereas for designs in which the cases' intervention start points were selected randomly without replacement, the restricted-Marascuilo-Busk test was conducted. For each test, the obtained test statistic was based on the appropriate mean difference. The Type I error (when $d=0$ ) or power (when $d>0$ ) was estimated for each test for each of the conditions by determining the proportion of the 10,000 simulated studies where the test led to a one-tailed $p$-value of .05 or less.

Results and Discussion

Type I error control. The results are summarized in the two panels of Figure 6. As in Investigation 1, Type I error control was adequate with all five procedures, with average empirical as ranging from .0475 (Koehler-Levin procedure with two potential intervention start points) to .058 (modified Revusky procedure with three potential intervention start points), both for the $N=4$ simulations in Panel $\mathrm{A}$.

Power. As may be seen in the two panels of Figure 6 ( $N=4$ and 5, respectively), both the Koehler-Levin and the restricted Marascuilo-Busk procedures are uniformly more powerful than the modified Revusky procedure, with little or no power difference between the former two procedures. At their most extreme (with an effect size given by $d=1.5$ ), the power advantages that the Koehler-Levin and restricted Marascuilo-Busk procedures exhibit over the modified Revusky procedure amount to about .10 for $N=4$ and .07 for $N=5$.

At the same time, it should not be forgotten that the results of Investigation 1 documented that, for $N>4$, the modified Revusky procedure has power qualities that are far superior to those of the original Revusky (1967) procedure (see Figures 4 and 5). This is an important finding insofar as over the years the original procedure continues to surface as a 
nonparametric analysis tool for multiple-baseline data (e.g., Brewer \& White, 1994; Crowe, Norris, \& Hoffman, 2004; Markham, Porter, \& Ball, 2011). With $N=4$ cases and an autocorrelation of .30 , the power similarities of the Koehler-Levin procedure (for either $k=2$ or 3 potential intervention start points) and the restricted Marascuilo-Busk procedure (for initial $k=$ 10) in our 20-observation series are quite compatible with what Ferron and Sentovich (2002) found when comparing the Koehler-Levin procedure (for $k=2$ ) and the original (unrestricted) Marascuilo-Busk procedure (for $k=11$ ) in a 19-observation series. We return to a further examination of the modified Revusky procedure in Investigation 4.

An initially surprising result is that for both the Koehler-Levin and modified Revusky procedures, the power benefits are impressive when increasing the number of potential intervention points from one to two, but hardly noticeable when further increasing the number of potential intervention start points to three, even with $N=4$ participants. An additional simulation that we conducted (not reported here) with $N=6$ cases confirms this conclusion. To gain a better understanding of the suggestion that there appear to be no power increases as the number of intervention start points increases beyond two, the present authors are currently investigating the combined "start-point/stagger" issue in a carefully controlled fashion. Preliminary results indicate that comparable power increases can be produced for a given randomization-test procedure by increasing either the number of potential intervention start points or the amount of stagger desired-a phenomenon that cannot be discerned unless one of these factors is held constant while the other is systematically manipulated.

\section{Investigation 3: Comparison of Three Procedures With Either Fixed or Randomly Selected Intervention Start Points}

In this investigation we probe further into the Type I error and power characteristics of a newly adapted multiple-baseline randomization test, the within-case restricted Marascuilo-Busk procedure. We do so in relation to both the Koehler-Levin procedure and its derivative 
Wampold-Worsham procedure when a fixed intervention start point is specified in advance. Specifically, here we examine: (1) the restricted Marascuilo-Busk randomization test for which intervention start-point selection is restricted to ensure between-case intervention start-point staggers of either at least one or at least two observations; (2) the Koehler-Levin procedure with either two or three potential intervention start points per case; and (3) the Wampold-Worsham procedure with fixed between-case intervention start-point staggers of three observations. The rationale for extending the Marascuilo-Busk procedure to an "at least a two-observation between-case stagger" was primarily to allow for a more-equal intervention start- point stagger among the $N$ cases. Consider, for example, an $N=4,20$-observation multiple-baseline design with 10 acceptable intervention start points, from just prior to Observation 6 through just prior to Observation 15. With the "at least one observation between-case stagger" requirement of Investigation 3, the worst-possible unequal-stagger scenario results would include the situations where the four randomly selected intervention start points are either (a) $\mathrm{O}_{6}, \mathrm{O}_{7}$ (a oneobservation stagger), $\mathrm{O}_{14}$ (seven observations), $\mathrm{O}_{15}$ (one observation); and (b) $\mathrm{O}_{6}, \mathrm{O}_{7}, \mathrm{O}_{8}$ (each a one-observation stagger) and $\mathrm{O}_{15}$ (seven observations). In contrast, with the "at least two observations" requirement, the worst-case scenarios would include (a) $\mathrm{O}_{6}, \mathrm{O}_{8}$ (a twoobservation stagger), $\mathrm{O}_{13}$ (five observations), $\mathrm{O}_{15}$ (two observations); and $(b) \mathrm{O}_{6}, \mathrm{O}_{8}, \mathrm{O}_{10}$ (each a two-observation stagger), $\mathrm{O}_{15}$ (five observations). In comparison to the former two stagger scenarios, the latter two do not seem as dramatically unequal, which should make them more appealing to multiple-baseline researchers (see also Koehler \& Levin, 1998, p. 209). ${ }^{2}$ Importantly, given this "at least two observations" specification, the restricted MarascuiloBusk procedure is no longer associated with a total of $k ! /(k-N) !=k \times(k-1) \times(k-2) \times \ldots \times(k-N+1)$ possible randomization outcomes, as in Investigation 2, but rather a reduced number, namely, 
$N !\left(\begin{array}{c}k-N+1 \\ N\end{array}\right)=(k-N+1) ! /(k-2 N+1) !$, with the requirement that $k \geq 2 N-1$. So, for example, given $N=$ 4 cases and 10 acceptable potential intervention points, whereas with an "at least one observation" requirement the total number of possible outcomes is $10 ! /(10-4) !=5,040$, with an "at least two observations" specification the total number is reduced to $4 !\left(\begin{array}{l}7 \\ 4\end{array}\right)=840$.

It should be noted that with four cases and 10 potential intervention start points, with the just-described restricted Marascuilo-Busk procedure it would also be possible to specify a stagger of "at least three observations" for all cases. For that particular specification, the previously presented formulas extend to $(k-2 N+2) ! /(k-3 N+2) !$, along with the requirement that $k$ $\geq 3 N-2$. Moreover, to specify a stagger of "at least s observations," the general formula is:

$$
\frac{[k-(s-1) N+(s-1)] !}{[k-s N+(s-1)] !}
$$

with the requirement that $k \geq s(N-1)+1$.

Applying an "at least 3 observations stagger" stipulation to the present $N=4, k=10$ example would therefore yield a total of $(10-8+2) ! /(10-12+2) !=4 ! / 0 !=4 !=24$ possible outcomes, which reduces the above total number of "at least two observations stagger" of 840 to the much smaller total of 24 when an "at least three observations stagger" is desired. From a practical standpoint, in this "at least three observations stagger" situation it should be a simple matter for a researcher to identify the only set of four start points between $\mathrm{O} 6$ and $\mathrm{O} 15$ that can be permissibly assigned to the four cases (namely $06,09,012$, and 015 ), for no other set fits the requirements of this example. The 24 possible stagger orders within the permissible set are listed and cases are randomly assigned to one of those orders. For example, one order would be that first case is randomly assigned to $\mathrm{O} 6$, the second to $\mathrm{O} 9$, the third to $\mathrm{O} 12$, and the fourth to O15. Alternatively, a different order of the permissible set might result in the first case having been randomly assigned to 012 , the second to 09 , the third to 015 , and the fourth to 06 . It is 
worth noting that for this particular $N=4$ example, the "at least three observations stagger" restricted Marascuilo-Busk design has been restricted to the point at which it becomes equivalent to the Wampold-Worsham design and thus the two respective powers are the same.

At the same time, this "at least three observations stagger" example unfortunately leaves a researcher in an unenvious "power" position, in that there now would be only a single opportunity for detecting a statistically significant multiple-baseline effect (as is also true for the Wampold-Worsham procedure). This is because only the single most extreme mean-difference outcome in the randomization distribution will produce a one-tailed $p$-value $(1 / 24=.042)$ that is less than a predefined $\alpha$ of .05. For that reason alone, we cannot in good conscience recommend an "at least three observations stagger" application of the restricted MarascuiloBusk procedure to analyze the data for the present $N=4, \mathrm{k}=10$ multiple-baseline design. Some combination of a decrease in the desired amount of stagger (e.g., from three to two observations), an increase in the size of the potential intervention start-point interval (e.g., from 10 to 16 observations), and an increase in the number of cases (e.g., from 4 to 5) would give this hypothetical study a much-needed power boost.

Method

As in the previous two investigations, we considered a four-case $(N=4), 19$-observation design and a five-case $(N=5)$, 22-observation design. For the former design, the restricted Marascuilo-Busk intervention start points (either with an "at least one" or an "at least two" between-cases stagger requirement) were randomly selected from the interval $\{6,15\}$; and for the latter design, the restricted Marascuilo-Busk intervention start points were randomly selected from the interval $\{6,18\}$. For convenience, certain comparison results associated with the Wampold-Worsham and Koehler-Levin procedures were obtained from Investigations 1 and 2. 
Unlike the two previous investigations, we also considered a three-case design $(N=3)$ with 16 observations per case. For this design, the Koehler-Levin procedure with two and three potential intervention start-point procedures had start points that were randomly selected from $\{6,7\},\{9,10\}$ and $\{12,13\}$ and $\{6,7,8\},\{9,10,11\}$, and $\{12,13,14\}$, respectively; and the two restricted Marascuilo-Busk procedures had intervention start points that were randomly selected from the interval $\{6,12\}$. Time-series data were generated for each case using the same method as in Investigations 1 and 2. The autocorrelation was set to .30 for all simulations and the effect sizes were varied from 0 to 4 in increments of .50. For each condition, data for 10,000 "studies" were collected.

Results and Discussion

The results of this investigation are presented in the three panels of Figure 7 for $N=3,4$, and 5 , respectively.

Type I error control. Average Type I errors ranged from .042 to .052 and so once again, they were well controlled by all test procedures for the number of potential intervention start points and amounts of stagger that were examined.

Power. It is clear from Figure 7 (and particularly for Ns of 3 and 4) that generally the randomized intervention start-point Koehler-Levin procedure and the restricted Marascuilo-Busk procedure are both more powerful than the fixed start-point Wampold-Worsham procedure based on a between-case stagger of three observations. That is true whether the former $(\mathrm{KL})$ is based on either two or three potential start points and the latter (MB-R) is based on a minimum stagger of either one or two observations. The results also indicate that: (1) for the particular $N$ $=3$ configurations investigated here, the Koehler-Levin procedure based on three potential start points is the most powerful and the Wampold-Worsham procedure has no power (because with $N=3$ the most extreme significance probability is $1 / 3 !=1 / 6=.167$ and so there is no chance for rejecting the null hypothesis); (2) for $N=4$ and 5, the powers of the two Koehler-Levin variations 
and the two restricted Marascuilo-Busk variations are quite similar; and (3) for $N=5$, the Wampold-Worsham procedure acquires power that rivals what is produced by the four other tests that were examined.

From a practical perspective, a multiple-baseline researcher's preference for selecting the restricted Marascuilo-Busk procedure based on a stagger of at least two observations over the same procedure based on a stagger of at least one observation might come down to the researcher's desire to have more equal-sized between-case staggers, as occurs with the Koehler-Levin procedure. This issue will be returned to in the General Discussion, where similar practical considerations will be discussed with respect to a researcher deciding which of the two procedures to adopt.

\section{Investigation 4: Comparison of Five Procedures With Respect to Alternative Intervention Effect Types}

In all of the previous investigations, we compared the Type I errors and powers of various multiple-baseline randomization tests (some old, some new) in situations where the intervention effect was modeled to be immediate and abrupt. ${ }^{3}$ In the present investigation, we extend these comparisons to situations in which the effect is modeled to be either delayed and abrupt or immediate and gradual. Specifically, here we examine the comparative sensitivities of the Wampold-Worsham, Koehler-Levin, restricted Marascuilo-Busk, original Revusky, and modified Revusky procedures to these two effect types.

Method

We considered multiple-baseline designs with the number of cases being four or five, and the series lengths being 19 for $N=4$ cases and 22 for $N=5$ cases. The potential start points for the interventions were either set in advance to $6,9,12$, and 15 for the $N=4$ designs, $6,9,12,15$, and 18 for the $N=5$ designs, or selected randomly. Random selection of intervention start points followed either the Koehler-Levin procedure or the restricted 
Marascuilo-Busk procedure. For the former, the selection was from either two or three potential intervention start points per case. For the $N=4$ design with two potential intervention start points per case, the start points were selected from $\{6,7\},\{9,10\},\{12,13\}$ and $\{15,16\}$, respectively. For the three-potential intervention start-point variation of the $N=4$ design, the start points were selected from $\{6,7,8\},\{9,10,11\},\{12,13,14\}$ and $\{15,16,17\}$. For $N=5$, we added the potential start-point set of $\{18,19\}$ and $\{18,19,20\}$ to the $N=4$ and $N=5$ designs, respectively, as was done in Investigation 2. With the restricted Marascuilo-Busk procedure, for the $N=4$ design the interventions were chosen without replacement from the interval 6 to 15 , inclusive, and for the $N=5$ design from the interval 6 to 18 , inclusive.

Time-series data were generated for each case using error generation methods that paralleled those of the previous investigations. The autocorrelation set to .30 but the effect series was coded differently. For immediate gradual effects, the effect series was coded to have values of 0 for the elements corresponding to baseline observations, $1 / 3 d$ for the element corresponding to the first intervention observation, $2 / 3 d$ for the element corresponding to the second intervention observation, and $d$ for all remaining elements in the effect series. For delayed-by-one-observation abrupt effects, the effect series was coded as 0 for elements corresponding to baseline observations, 0 for the first element during intervention, and $d$ for all remaining elements in the effect series. For delayed-by-two-observation abrupt effects, the effect series was coded as 0 for elements corresponding to baseline observations, 0 for the first two elements during intervention, and $d$ for all remaining elements in the effect series. As in the previous investigations, values of $d$ were varied from 0 to 4.0 in increments of .5 , and the observed sequence was obtained by adding the error series to the effect series.

By crossing the number of cases $(N=4,5)$, by the number of possible start points per case $(k=1,2,3)$, by the effect size ( $d=0$ to 4 , in increments of .5$)$, by the type of effect (immediate gradual, delayed-by-one-observation abrupt, or delayed-by-two-observations 
abrupt), 162 unique conditions were formed. For each condition, data for 10,000 "studies" were simulated for each randomized design and then analyzed with the corresponding randomization test. More specifically, for designs with random order and a single fixed start point the Wampold-Worsham, Revusky, and modified Revusky tests were conducted, for designs with random order and randomly selected start points the Koehler-Levin and modified Revusky tests were conducted, and for designs where start points were selected randomly without replacement the restricted-Marascuilo-Busk test was conducted. For each test the obtained test statistic was based on the mean difference between intervention and baseline observations. The Type I error (when $d=0$ ) or power (when $d>0$ ) was estimated for each test for each of the 162 conditions by determining the proportion of the 10,000 simulated studies where the test led to a one-tailed $p$-value of .05 or less.

Results and Discussion

The results are summarized in Figures 8 and 9 , for $N=4$ and $N=5$, respectively, where in each figure are displayed Type I error and power for effects that are delayed by one observation and abrupt (Panel A), effects that are delayed by two observations and abrupt (Panel B), and effects that are immediate and gradual (Panel C).

Type I error control. As in the previous investigations, the Type I error control associated with all procedures was acceptable here, with averages ranging from .039 to .058 for the $N=4$ designs and from .038 to .056 for the $N=5$ designs.

Power. First, in striking contrast to the previous investigations' powers associated with the immediate abrupt effects of Investigation 3's Figure 7, the powers associated with the present investigation's effect types take a serious "hit" for all procedures—the most dramatic of which are the powers for two-observations-delayed abrupt effects (Panel B in Figures 8 and 9). Consider, as an example, the Koehler-Levin procedure based on $N=4$ cases and $k=3$ potential intervention start points per case. The power is .81 to detect an immediate abrupt 
effect of 1.5 (Panel B of Figure 7), whereas the powers are only .38, .32, and .09, respectively, to detect a $d=1.5$ immediate gradual effect (Panel C of Figure 8), a delayed-by-oneobservation abrupt effect (Panel A of Figure 8), and a delayed-by-two-observations abrupt effect (Panel B of Figure 8). A similar precipitous drop in power occurs when $N=5$, where they are $.92, .65, .60$, and .24 , respectively (see Panel C of Figure 7 and Figure 9 ). It can be ascertained from Figures 7, 8, and 9 that although most of the randomization-test powers investigated here have respectable power for detecting immediate abrupt effects of a moderate size, they are inadequate for detecting other effect types of the same magnitude. This conclusion should be taken especially seriously for effects that emerge in a gradual (rather than in an abrupt) fashion.

Second, despite the power declines seen in Figures 8 and 9 for all test procedures, differences among them are still apparent. Generally speaking, when an immediate gradual effect was produced (Panel C in Figures 8 and 9) the within-case procedures (WampoldWorsham, Koehler-Levin, and restricted Marascuilo-Busk) fared better than the between-case procedures (Revusky and modified Revusky). Similarly, for abrupt effects delayed by one observation, and especially when $N=5$, the within-case procedures (headed by WampoldWorsham) were more powerful than the between-case procedures, with the modified Revusky procedure based on three potential intervention start points being distinctly inferior (Panel A of Figure 9). Finally, for abrupt effects delayed by two observations, there was little to choose among the various procedures, as they were all lacking in power (Panel B of Figures 8 and 9). If a "best among the worst" choice had to be made for detecting such effects, however, it would be for the within-case Wampold-Worsham procedure when $N=5$, even though for very large effect sizes ( $d$ s of 3.5 and 4 ) that procedure is actually surpassed by the between-case modified Revusky procedure with a single fixed intervention start point (Panel B of Figure 9).

These results clearly indicate that if a researcher is "off" in specifying the immediacy and/or type of effect expected of the intervention (i.e., if there is a mismatch between the 
expected and obtained effect type), then none of the present test procedures, as formulated here, will do an adequate job. The "as formulated here" addition is critical however, in that it is possible for a researcher to adapt any of the test procedures to take advantage of different effect types. For example, for all procedures examined here, with a two-observations-delayed abrupt effect expected, the researcher would simply define (on an a priori basis) the B-A phasemean difference for each case to start with the B phase's third outcome observation. An adaptation of this kind was not incorporated into the present simulations because if it were and if the expected effect were in fact modeled, the results would have perfectly replicated those of the present simulations for which immediate abrupt effects were modeled to be consistent with all of the procedures' tests of B-A phase-mean differences. Fortunately, for multiple-baseline researchers the adaptation is readily managed in Gafurov and Levin's (2015) ExPRT randomization-test package by simply specifying that the first post-intervention observation (for a one-observation-delayed effect) or the few observations is/are "missing." ExPRT will ignore the missing data and calculate within-phase means without it/them, thereby resulting in means that are "delayed" by one or a few observations.

For situations in which a gradual A-to-B-phase change in level (means) is expected (as was modeled here in Investigation 4), a randomization test statistic can be formulated in terms of a difference in the slope associated with the two phases (see, for example, Koehler and Levin, 1998, p. 215; and Levin, Evmenova, \& Gafurov (2014, p. 190). A simulation study examining the statistical properties of "slope" tests, as well as those for randomization tests of variability differences, is now in progress (Levin, Ferron, \& Gafurov, 2015). In short, to benefit from any of the present randomization-test procedures, the researcher must have a reasonably good knowledge base regarding the type of intervention effect to be expected. This same caveat applies equally forcefully to other single-case statistical strategies, such as hierarchical 
linear modeling (HLM) approaches (see, for example, Ferron, Moeyaert, Van den Noortgate, \& Beretvas, 2014).

\section{General Discussion}

Based on the present four-investigation series in which several different randomization statistical tests for single-case multiple-baseline designs were compared, we offer the following general conclusions and recommendations.

With precious few exceptions, the newer class of randomized intervention start-point models exhibited superior statistical power qualities relative to the traditional single fixed-point models, and often decisively so. In addition, of the three procedures compared in Investigation 1 (for which $N=4$ and 5), the modified Revusky procedure based on a single start point outperformed the original Revusky procedure, and the single start-point Wampold-Worsham (1986) procedure distinguished itself as the most powerful of the three. Consequently, the nearly 50-year-old original Revusky (1967) test should be abandoned in favor of the more recently developed Koehler-Levin (1998), restricted Marascuilo-Busk, and modified Revusky models, the latter two of which were introduced and examined in earnest here. It would be difficult for a single-case researcher to justify continued use of the Revusky test, other than on grounds of its computational simplicity: namely, that no special statistical software package is required to perform the test's calculations, other than perhaps a basic spreadsheet program.

Of the various randomized start-point models available, the results of Investigation 2 suggest that the Koehler-Levin and restricted Marascuilo-Busk procedures are superior to the modified Revusky procedure. The results of Investigation 3 additionally indicate that both the Koehler-Levin procedure based on three potential start points and the restricted MarascuiloBusk procedure based on at least a one-observation stagger outperform the WampoldWorsham procedure based on a stagger of three observations in $\mathrm{N}=3$ multiple-baseline studies (where the Wampold-Worsham test has no power when $\alpha=.05$ ) as well as in $N=4$ studies. 
When $N>4$, however, it appears that all three procedures are associated with similar powers and so, as is discussed below, a choice among them must be made on other than statistical grounds. Considerations about whether to apply the Koehler-Levin procedure with two or three potential intervention start points or the restricted Marascuilo-Busk procedure with at least a one, two, or more observation(s) between-case stagger are being formulated in the earlier mentioned start-point/stagger simulation study of Ferron \& Levin (2015).

The foregoing conclusions and recommendations are derived from simulations in which the intervention effects were modeled to be immediate and abrupt. In Investigation 4, the powers for all procedures were drastically reduced for effect types that were not so typical. Yet, the within-case Koehler-Levin and restricted Marascuilo-Busk procedures, and especially the fixed start-point Wampold-Worsham procedure, continued to be more sensitive in detecting both abrupt one-observation-delayed and immediate gradual effects than the between-case Revusky variations (see also Tyrrell, Corey, Feldman, and Silverman, 2013, who have recently proposed an alternative power-increasing strategy for the Wampold-Worsham procedure, based on combining the test results of two separately conducted $N=4$ multiple-baseline studies).

Even though the fixed start-point modified Revusky procedure emerged as the "victor" for two-observation delayed abrupt effects, its "absolute power" was so low that the victory should be encoded as a Pyrrhic one. As was noted earlier, a researcher can anticipate these (or other) alternative effect types on the basis of prior research or theory-based predictions, and then prepare for them by adapting the randomization test (or computer software) to match the characteristics of the anticipated effect type, their associated powers will increase.

\section{A Few Operational Issues to Consider}

We conclude this article with an extended discussion of a few "operational issues" that will confront researchers when making decisions about which of the various multiple-baseline 
randomization-test procedures to consider adopting, or even whether to adopt any of them at all.

Restricted Marascuilo-Busk procedure. We reiterate that both the randomized start-point Koehler-Levin procedure and the restricted Marascuilo-Busk procedure have much to recommend themselves in the present multiple-baseline design context. The Koehler-Levin procedure is straightforward with respect to its logic and implementation, both of which have been discussed in detail when the procedure was originally introduced (Koehler \& Levin, 1998). The same is not true of the restricted Marascuilo-Busk procedure and so here we offer some advice concerning the appropriate way in which it is to be implemented.

As has been noted throughout this article, the original Marascuilo-Busk (1988) procedure is simple to execute in that it involves a sampling-with-replacement process for all cases. That is, an acceptable intervention start-point interval is specified for all cases and their start points are independently sampled from that interval. Two unfortunate consequences of the process, however, are that: (1) stagger is not controlled for in any way, leading to the possibility that the amount of between-case stagger has the potential to be markedly unequal; and (2) even worse, the possibility exists that two (or more) cases could end up with exactly the same intervention start point, which flies in the face of a multiple-baseline design. Our present restricted Marascuilo-Busk procedure based on an "at least one observation stagger" was devised to deal with the latter consequence and the procedure based on an "at least two observations stagger" was devised to deal more effectively with the former consequence.

Consider, as we did earlier in the introduction to Investigation 3 , an $N=4$ design with 20 total observations and 10 potential intervention start points that are designated prior to the study's commencement. A researcher can implement the "at least one observation stagger" Marascuilo-Busk procedure appropriately in three (or more) equivalent ways. One way is to randomly sample intervention start points for each case successively without replacement, 
starting with 10 possibilities for the first case, then moving on to 9 for the second case, 8 for the third case, and 7 for the fourth case. A second way is to follow the original Marascuilo-Busk sampling-with-replacement process with the stipulation that if exactly the same intervention start point is selected for a successive case, then that start point is ignored and a second start point is sampled, and similarly for the third and fourth cases, with the process continuing in that fashion until the four cases have all been assigned different start points. A third way, and one that is incorporated into the EXPRT randomization test package (Gafurov \& Levin, 2015), is for the researcher to draw repeated samples of four intervention start points from within the acceptable interval until landing on a sample in which all four start points differ. For an $N=4$ design, this process typically would require two or three iterations at most. All of these sampling variations guarantee that no two cases will be assigned the same intervention start point.

It must be stressed that whichever four start points are selected during the initial selection process must be included in the study's design, regardless of how equally spaced they may appear to be with respect to the amount of between-case stagger (i.e., whether they are in the "reasonable" positions 3, 5, 7, and 9 or in the "not so reasonable" positions 1, 2, 9, and 10). Somewhat akin to Tom Hanks' classic "consoling" declaration "There's no crying in baseball" in the wonderful movie, A League of Their Own, there can be no "do overs" of the sampling process here. Any disturbance of this single effort's outcomes will result in significance probabilities that are not compatible with a "random" sampling model and such probabilities will therefore be incorrect.

The "at least two observations stagger" restricted Marascuilo-Busk procedure was devised because with it, there generally will be a greater chance of obtaining a more equal between-case stagger. Implementing that version of the procedure in accordance with its underlying probabilistic structure is decidedly more complicated, and likely more timeconsuming without the assistance of specially developed computer software. Without a special 
computer program, there are again three (or more) ways for a researcher to proceed. First, for a given number of cases and a predetermined acceptable intervention start-point interval, the researcher could repeatedly randomly sample start points as in the "at least one observation" stagger situation, until landing on a start-point configuration that satisfies the "at least two observations" stagger criterion for all cases. Second, the researcher could rely on a computer package such as ExPRT to draw samples of size $N$ until a sample is produced that satisfies the "at least two observations stagger" requirement. Third, and especially for a small number of cases and potential start points, the researcher could systematically list all possible permutations of the cases within start-point positions and randomly sample a single permutation from that list. In our $N=4,10$ potential start-point example, the total number of permutations is the earlier calculated 840, which would be an onerous task for most humans. Yet, with a reduced number of potential start points, say 7 , the total number of permutations would be drastically reduced, to 24 , which is a number that most humans could be expected to manage.

An alternative, more readily implemented, "successively restricted" sampling procedure-not examined here and similar to a suggestion made by Koehler and Levin (1998, Footnote 7)—can be formulated to achieve more equal between-case staggers. With this procedure, the researcher would randomly select the first case's intervention start point from the usual pre-specified acceptable interval. After seeing where that start point falls, the researcher would specify a new acceptable interval from which the second case's start point is to be selected. That new range would be specified with the desired amount of stagger taken into consideration, and the same process would continue for the remaining cases. So, for the preceding example with $N=4$ cases, 20 total observations, an acceptable interval consisting of the middle 10 observations, and a specified between-case stagger of at least two observations, the researcher would randomly select an intervention start point for the first case from the 10observation interval $\left(\mathrm{O}_{6}-\mathrm{O}_{15}\right)$. Suppose that the selected start point turns out to be just prior to 
Og. To ensure at least a two-observation stagger for the second case, that case's acceptable interval would include $\mathrm{O}_{6}$ and $\mathrm{O}_{7}$, along with $\mathrm{O}_{11}-\mathrm{O}_{15}$. If $\mathrm{O}_{6}$ were selected for the second case, to ensure at least a two-observation stagger for the third case the acceptable Interval would include $\mathrm{O}_{11}-\mathrm{O}_{15}$. With $\mathrm{O}_{15}$ randomly selected for that case, to ensure at least a two-observation stagger for the final case the acceptable interval would include $\mathrm{O}_{11}-\mathrm{O}_{13}$. With $\mathrm{O}_{11}$ randomly selected for that case, the final stagger positions for the four (reordered) cases would be: $\mathrm{O}_{6}$, $\mathrm{O}_{9}, \mathrm{O}_{11}$, and $\mathrm{O}_{15}$, with between-case staggers ranging from two to four observations.

A precaution to keep in mind with this successively restricted procedure is that when the ratio, consisting of the number of potential intervention start points in the initial interval $(k)$ relative to twice the number of cases $(2 N)$, is small (i.e., when $k$ is very close to $2 N$ in size), without careful monitoring on the part of the researcher the likelihood increases that the sequential-sampling methods just described will fail to produce a permissible start-point set. Take, for example, a situation in which 5 cases are to be assigned to 9 different start points that are separated by at least two observations. Now suppose that the first case is randomly assigned to receive start point 4. Although it appears that there are still plenty of "open" start points for the remaining four cases, it turns out that there are not enough remaining permissible start points. With an initial assignment of start point 4 to the first case (or for this example, any even-numbered start point), there is no configuration for which all 5 cases can be separated by at least two observations. In the present example with the first case assigned to position 4, the next three cases could be assigned to positions 2,6 , and 8 , but that is the end of the road. With the present total of only 9 potential start points, there is no other start point for the fifth case that is at least two observations away from each of the others. As was indicated briefly at the outset, for this procedure to produce a usable set of start points the first time it is implemented under small $k / 2 N$ ratio situations, the researcher would be wise to map out in advance all possible permutations of the permissible start-point spacings and then randomly select one permutation 
from that set. For the above example, it can be readily seen that the only configuration that will "work" is when the start points selected occupy positions $1,3,5,7$, and 9 , and permuting those five positions results in $5 !=120$ permissible possible outcomes.

Different series lengths for the different cases. Finally, let us revisit the issue of administering series of different lengths to the different cases. This is another critical pragmatic research consideration, which may arise when it is either not convenient or not possible (economically or otherwise) for the researcher to continue implementing an initial case's intervention (B) phase throughout the entirety of the later cases' B phases. For example, the following situation is not uncommon in an $N=4$ multiple-baseline study with 25 total observations. Rather than providing 25 observations for all four cases, the researcher allows the first case's A phase to run from Observation 1 through Observation 5 and the B phase to run from $\mathrm{O}_{6}$ through $\mathrm{O}_{10}$ (10 total observations), the second case's $A$ and $B$ phases to run from $\mathrm{O}_{1}$ through $\mathrm{O}_{10}$ and $\mathrm{O}_{11}$ through $\mathrm{O}_{15}$, respectively (15 total observations), the third case's $\mathrm{A}$ and $\mathrm{B}$ phases from $\mathrm{O}_{1}$ through $\mathrm{O}_{15}$ and $\mathrm{O}_{16}$ through $\mathrm{O}_{20}$, respectively (20 total observations), and the fourth case's $A$ and $B$ phases from $O_{1}$ through $\mathrm{O}_{20}$ and $\mathrm{O}_{21}$ through $\mathrm{O}_{25}$, respectively (25 total observations).

A researcher's decision to provide unequal numbers of observations for the $N$ different cases is perfectly acceptable, with some "restrictions," for the multiple-baseline procedures that involve no between-case permutations (specifically, for the restricted Marascuilo-Busk, Revusky, and modified Revusky procedures). For the restricted Marascuilo-Busk test, there are no constraints whatsoever on how long each case's A and B series should be because with that procedure no direct connection is needed between one case's series and another case's series. With the Revusky and modified Revusky procedures, however, partial inter-case connections are required on each step. On the first step of the analysis, all $N$ cases must be connected; on the second step, $N$-1 cases (all except the first case) must be connected; and so on. This 
means that if the cases are ordered temporally in terms of the shortest to longest baseline phase, each preceding case's B phase must continue at least through the following case's A phase, after which the preceding case's B phase is allowed to terminate. So, in the $N=4$ situation, the first case's B phase must continue at least through the second case's A phase, the second case's B phase must continue at least through the third case's A phase, and the third case's B phase must continue at least through the fourth case's A phase.

In contrast to the modest series length requirements for the procedures just discussed, more restrictive ones are attached to the Wampold-Worsham and Koehler-Levin procedures, both of which involve direct connections among the cases (namely, data permutations involving all $N$ cases) throughout the study. For that reason, with those procedures all cases' B series must continue at least through each of the other series' potential start-point intervals. ${ }^{4}$

There are both statistical and practical reasons for our providing the preceding specifications. Concerning the former, if the specifications are not adhered to, then a randomization test applied to the study's outcome data is at best questionable and at worst invalid. Concerning the latter, although we found here that the Wampold-Worsham and KoehlerLevin procedures generally yielded the greatest power for equal series length designs, in situations where the different cases' post-intervention series are terminated at different times (i.e., when not all cases are able or are allowed to continue the series through the final case's intervention start point) then the "equal series length" randomization-test procedures cannot be applied. Fortunately, however, both the restricted Marascuilo-Busk and modified Revusky procedures exhibit power that in many instances is not appreciably lower than that of the "equal series length" procedures. 


\section{References}

Barton, E. E., \& Reichow, B. (2012). Guidelines for graphing data with Microsoft® Office $2007^{\mathrm{TM}}$, Office $2010^{\mathrm{TM}}$, and Office for Mac ${ }^{\mathrm{TM}} 2008$ and 2011. Journal of Early Intervention, 34, 129-150.

Brewer, N., \& White, J. M. (1994). Computerized handwriting instruction with severely mentally handicapped adults. Journal of Intellectual Disability Research, 38, 37-44.

Busk, P. L., \& Serlin, R. C. (1992). Meta-analysis for single-case research. In T. R. Kratochwill \& J. R. Levin (Eds.), Single-case research design and analysis: New developments for psychology and education (pp. 187-212). Hillsdale, NJ: Erlbaum.

Campbell, D. T., \& Stanley, J. C. (1966). Experimental and quasi-experimental designs for research. Chicago, IL: Rand McNally.

Crowe, L. K., Norris, J. A., \& Hoffman, P. R. (2004). Training caregivers to facilitate communicative participation of preschool children with language impairment during storybook reading. Journal of Communication Disorders, 37, 177-196.

Edgington, E. S. (1975). Randomization tests for one-subject operant experiments. Journal of Psychology, 90, 57-58.

Edgington, E. S. \& Onghena, P. (2007). Randomization tests (4 $4^{\text {th }}$ ed.). Boca Raton, FL: Chapman \& Hall.

Ferron, J., Farmer, J., \& Owens, C. (2010). Estimating individual treatment effects from multiplebaseline data: A Monte Carlo study of multilevel modeling approaches. Behavior Research Methods, 42, 930-943.

Ferron, J. M., \& Levin, J. R. (2014). Single-case permutation and randomization statistical tests: Present status, promising new developments. In T. R. Kratochwill \& J. R. Levin (Eds.), Single-Case Intervention Research: Statistical and Methodological Advances (pp. 153183). Washington, DC: American Psychological Association. 
Ferron, J. M., Moeyaert, M., Van den Noortgate, W., \& Beretvas, S. N. (2014). Estimating causal effects from multiple-baseline studies: Implications for design and analysis. Psychological Methods, 19, 493-510.

Ferron, J., \& Sentovich, C. (2002). Statistical power of randomization tests used with multiplebaseline designs. Journal of Experimental Education, 70, 165-178.

Ferron, J., \& Ware, W. (1995). Analyzing single-case data: The power of randomization tests. Journal of Experimental Education, 63, 167-178.

Gafurov, B. S., \& Levin, J.R. (2015, May). ExPRT (Excel7 Package of Randomization Tests): Statistical Analyses of Single-Case Intervention Data (Version 2.0). Downloadable from https://code.google.com/p/exprt/.

Horner, R. H., \& Odom, S. L. (2014). Constructing single-case research designs: Logic and options. In T. R. Kratochwill \& J. R. Levin (Eds.), Single-case intervention research: Methodological and statistical advances (pp. 27-51). Washington, DC: American Psychological Association.

Hwang, Y., Levin, J.R., \& Johnson, E.W. (in press). Pictorial mnemonic-strategy interventions for children with special needs: Illustration of a multiply randomized single-case crossover design. Developmental Neurorehabilitation. Online version available at http://dx.doi.org/10.3109/17518423.2015.1100689.

Koehler, M. J., \& Levin, J. R. (1998). Regulated randomization: A potentially sharper analytical toll for the multiple-baseline design. Psychological Metho Hwang Y, Levin JR, Johnson EW. Pictorial mnemonic-strategy interventions for children with special needs: Illustration of a multiply randomized single-case crossover design. Developmental Neurorehabilitation 2016. Online version available at http://dx.doi.org/10.3109/17518423.2015.1100689ds, 3, 206-217. 
Kratochwill, T. R., Hitchcock, J. H., Horner, R. H., Levin, J. R., Odom, S. L., Rindskopf, D. M. \& Shadish, W. R. (2013). Single-case intervention research design standards. Remedial and Special Education, 34, 26-38.

Kratochwill, T. R., \& Levin, J. R. (2010). Enhancing the scientific credibility of single-case intervention research: Randomization to the rescue. Psychological Methods, 15, 122144.

Kratochwill, T. R., Levin, J. R., Horner, R. H., \& Swoboda, C. M. (2014). Visual analysis of single-case intervention research: Conceptual and methodological issues. In T. R. Kratochwill \& J. R. Levin (Eds.), Single-case intervention research: Methodological and statistical advances (pp. 91-125). Washington, DC: American Psychological Association.

Levin, J. R. (1992). Single-case research design and analysis: Comments and concerns. In T. R. Kratochwill \& J. R. Levin (Eds.), Single-case research design and analysis: New developments for psychology and education (pp. 213-224). Hillsdale, NJ: Erlbaum.

Levin, J. R. (1994). Crafting educational intervention research that's both credible and creditable. Educational Psychology Review, 6, 231-243.

Levin, J. R., Evmenova, A. S., \& Gafurov, B. S. (2014). The single-case data-analysis ExPRT (Excel7 Package of Randomization Tests). In T. R. Kratochwill \& J. R. Levin (Eds.), Single-case intervention research: Methodological and statistical advances (pp.185219). Washington, DC: American Psychological Association.

Levin, J. R., Ferron, J. M., \& Gafurov, B. S. (2014). Improved randomization tests for a class of single-case intervention designs. Journal of Modern Applied Statistical Methods, 13(2), 2-52; retrievable from http://digitalcommons.wayne.edu/jmasm/vol13/iss2/2.

Levin, J. R., Ferron, J. M., \& Gafurov, B. S. (2015). Research in progress. University of Arizona, Tucson. 
Levin, J. R., \& Wampold, B. E. (1999). Generalized single-case randomization tests: Flexible analyses for a variety of situations. School Psychology Quarterly, 14, 59-93.

Marascuilo, L. A., \& Busk, P. L. (1988). Combining statistics for multiple-baseline AB and replicated ABAB designs across subjects. Behavioral Assessment, 10, 1-28.

Markham, P. T., Porter, B. E., \& Ball, J. D. (2011). Effectiveness of a program using a vehicle tracking system, incentives, and disincentives to reduce the speeding behavior of drivers with ADHD. Journal of Attention Disorders, 17, 233-248.

Parker, R. I., Vannest, K. J., \& Davis, J. L. (2014). Non-overlap analysis for single-case research. In T. R. Kratochwill \& J. R. Levin (Eds.), Single-case intervention research: Methodological and statistical advances (pp. 127-151). Washington, DC: American Psychological Association.

Revusky, S. H. (1967). Some statistical treatments compatible with individual organism methodology. Journal of the Experimental Analysis of Behavior, 10, 319-330.

Siegel, S. (1956). Nonparametric statistics for the behavioral sciences. New York: McGraw-Hill. Tyrrell, P. N., Corey, P. N., Feldman, B. M., \& Silverman, E. D. (2013). Increased statistical power with combined independent randomization tests used with multiple-baseline design. Journal of Clinical Epidemiology, 66, 291-294.

Wampold, B., \& Worsham, N. (1986). Randomization tests for multiple-baseline designs. Behavioral Assessment, 8, 135-143. 


\section{Footnotes}

1. Gafurov and Levin's (2015) ExPRT program allows the user to specify either difference option (i.e., simple or relative).

2. The notion of a "minimum" stagger is introduced here because with the Koehler-Levin randomized intervention start-point procedure (i.e., when $k>1$ ), one cannot specify precisely in advance where each case's actual start point will occur. Consider, for example, a desired two-observation stagger between cases and an interval containing three potential intervention start points specified to include $\mathrm{O}_{6}-\mathrm{O}_{8}$ for Case 1 . With that interval and the "two-observation stagger" restriction, Case 2's start-point interval containing three potential intervention start points could not begin until $\mathrm{O}_{10}$ and would run through $\mathrm{O}_{12}$. Thus, the actual amount of stagger between Cases 1 and 2 could be as small as two observations $\left(\mathrm{O}_{9}\right.$ and $\left.\mathrm{O}_{10}\right)$ or as large as six observations $\left(\mathrm{O}_{7}\right.$ through $\left.\mathrm{O}_{12}\right)$.

3. Immediate abrupt effects have been modeled almost exclusively in previous Monte Carlo simulations involving single-case statistical procedures (see, for example, Levin, Ferron, \& Gafurov, 2014).

4. In addition, "missing data" represents a data-analysis issue that differs from that of the "unequal series lengths" issue. Although missing data can be handled by each of the procedures examined here, irreparable probability-calculation problems are created for all of the random intervention start-point models (restricted Marascuilo-Busk, KoehlerLevin, and modified Revusky) when data are missing within any of those procedures' potential start-point intervals (see also Levin, Evmenova, \& Gafurov, 2014, p. 192). 
Table 1. Multiple-Baseline Design for All Randomization-Test Examples: Three Classrooms (C) and 10 Time Periods (T) Subdivided into A and B Phases

$\begin{array}{llllllllll}T_{1} & T_{2} & T_{3} & T_{4} & T_{5} & T_{6} & T_{7} & T_{8} & T_{9} & T_{10}\end{array}$

\begin{tabular}{lll|lllllllll}
$C_{1}$ & $\mathrm{~A}$ & $\mathrm{~A}$ & $\mathrm{~B}$ & $\mathrm{~B}$ & $\mathrm{~B}$ & $\mathrm{~B}$ & $\mathrm{~B}$ & $\mathrm{~B}$ & $\mathrm{~B}$ & $\mathrm{~B}$
\end{tabular}

\begin{tabular}{llllll|llllll}
$C_{2}$ & $\mathrm{~A}$ & $\mathrm{~A}$ & $\mathrm{~A}$ & $\mathrm{~A}$ & $\mathrm{~A}$ & $\mathrm{~B}$ & $\mathrm{~B}$ & $\mathrm{~B}$ & $\mathrm{~B}$ & $\mathrm{~B}$
\end{tabular}

\begin{tabular}{llllllll|llll}
$C_{3}$ & $\mathrm{~A}$ & $\mathrm{~A}$ & $\mathrm{~A}$ & $\mathrm{~A}$ & $\mathrm{~A}$ & $\mathrm{~A}$ & $\mathrm{~A}$ & $\mathrm{~B}$ & $\mathrm{~B}$ & $\mathrm{~B}$
\end{tabular}

Note: Classrooms have been randomly assigned to the three rows of the design, with | representing the actual intervention start point for each classroom. 
Table 2. Data and Wampold-Worsham Partial Calculations for the Classroom Intervention Example

Actual Intervention Order (Classroom 1, 2, 3)

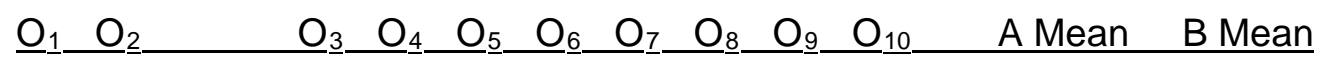

B-A Mean

\begin{tabular}{lll|lllllllllll} 
Classroom 1 & 4 & 3 & 5 & 7 & 6 & 8 & 7 & 8 & 9 & 7 & 3.500 & 7.125 & 3.625
\end{tabular}

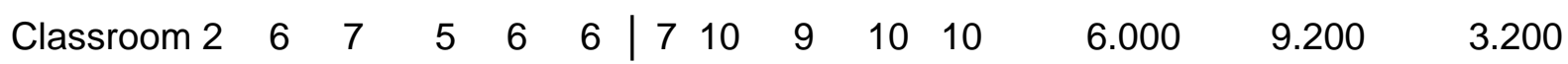

\begin{tabular}{llllllll|lllllll} 
Classroom 3 & 9 & 9 & 7 & 10 & 10 & 8 & 9 & 12 & 11 & 14 & $\underline{8.857}$ & 12.333 & 3.476 \\
\hline
\end{tabular}

Across Classrooms $\quad 6.119 \quad 9.553 \quad 3.434$

One Possible Alternative Intervention Order (Classroom 1, 3, 2)

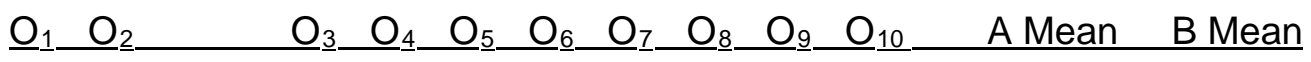
B-A Mean

\begin{tabular}{lll|lllllllllll} 
Classroom 1 & 4 & 3 & 5 & 7 & 6 & 8 & 7 & 8 & 9 & 7 & 3.500 & 7.125 & 3.625
\end{tabular}

\begin{tabular}{llllll|llllllll} 
Classroom 3 & 9 & 9 & 7 & 10 & 10 & 8 & 9 & 12 & 11 & 14 & 9.000 & 10.800 & 1.800
\end{tabular}

\begin{tabular}{llllllll|llllll} 
Classroom 2 & 6 & 7 & 5 & 6 & 6 & 7 & 10 & 9 & 10 & 10 & $\underline{6.714}$ & 9.667 & 2.953 \\
\hline
\end{tabular}

Across Classrooms $\quad 6.405 \quad 9.197 \quad 2.792$

Note: | indicates the actual intervention start point for each classroom. 
Table 3. Design, Data, and Marascuilo-Busk Partial Calculations for the Classroom Intervention Example

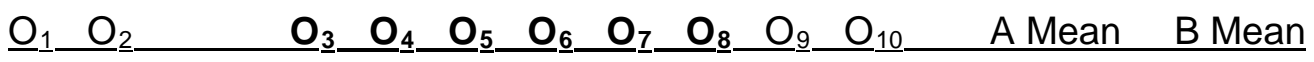

\begin{tabular}{|c|c|c|c|c|c|c|c|c|c|c|c|c|c|}
\hline Classroom 1 & 6 & 7 & 5 & 6 & 6 & 7 & 10 & 9 & 10 & 10 & 6.000 & 9.200 & 3.200 \\
\hline Classroom 2 & 9 & 9 & 7 & 10 & 10 & 8 & 9 & 12 & 11 & 14 & 8.857 & 12.333 & 3.476 \\
\hline Classroom 3 & 4 & 3 & 5 & 7 & 6 & 8 & 7 & 8 & 9 & 7 & 3.500 & 7.125 & 3.625 \\
\hline
\end{tabular}

$\underline{\text { Note: }}$ The bolded Os represent the six-alternative intervention start-point interval that was specified for each of the three classrooms. | indicates the actual intervention start point for each classroom. 
Table 4. Design, Data, and Koehler-Levin Partial Calculations for the Classroom Intervention Example

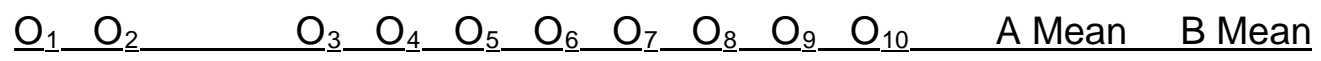

B-A Mean

\begin{tabular}{lll|lllllllllll} 
Classroom 1 & 4 & $\mathbf{3}$ & $\mathbf{5}$ & 7 & 6 & 8 & 7 & 8 & 9 & 7 & 3.500 & 7.125 & 3.625
\end{tabular}

\begin{tabular}{llllll|llllllll} 
Classroom 2 & 6 & 7 & 5 & 6 & $\mathbf{6}$ & $\mathbf{7}$ & 10 & 9 & 10 & 10 & 6.000 & 9.200 & 3.200
\end{tabular}

\begin{tabular}{llllllll|lllllll} 
Classroom 3 & 9 & 9 & 7 & 10 & 10 & 8 & 9 & 12 & 11 & 14 & $\underline{8.857}$ & 12.333 & 3.476 \\
\hline
\end{tabular}

Across Classrooms $\quad 6.119 \quad 9.553 \quad 3.434$

Note: The bolded values in the table represent the two-alternative intervention start-point interval that was specified for each classroom. | indicates the actual intervention start point for each classroom. 
Table 5. Design, Data, and Revusky Calculations for the Classroom Intervention Example

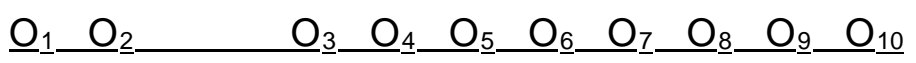

\begin{tabular}{lll|llllllll} 
Classroom 1 & 4 & 3 & 5 & 7 & 6 & 8 & 7 & 8 & 9 & 7
\end{tabular}

Classroom $2 \quad 6 \quad$\begin{tabular}{lllll|lllll} 
& 6 & 5 & 6 & 6 & 7 & 10 & 9 & 10 & 10
\end{tabular}

\begin{tabular}{llllllll|lll} 
Classroom 3 & 9 & 9 & 7 & 10 & 10 & 8 & 9 & 12 & 11 & 14
\end{tabular}

Step 1

\begin{tabular}{|c|c|c|c|c|}
\hline Mean & $\underline{\mathrm{A}=\mathrm{O}_{1}}: \underline{\mathrm{O}_{2}}$ & $\underline{\mathrm{B}=\mathrm{O}_{3}}: \underline{\mathrm{O}_{5}}$ & $\underline{B-A}$ & $\underline{\text { Rank }}$ \\
\hline Classroom 1* & 3.500 & 6.000 & 2.500 & 1 \\
\hline Classroom 2 & 6.500 & 5.667 & -0.833 & 3 \\
\hline Classroom 3 & 9.000 & 9.000 & 0.000 & 2 \\
\hline
\end{tabular}

Step 2

$\underline{\text { Mean }} \quad \underline{\mathrm{A}=\mathrm{O}_{1}}: \underline{\mathrm{O}_{5}} \underline{\mathrm{B}=\mathrm{O}_{6}}: \underline{\mathrm{O}_{7}} \quad \underline{\mathrm{B}-\mathrm{A}} \quad \underline{\text { Rank }}$

$\begin{array}{lllll}\text { Classroom } 2^{*} & 6.000 & 8.500 & 2.500 & 1\end{array}$

$\begin{array}{lllll}\text { Classroom } 3 & 9.000 & 8.500 & -0.500 \quad 2\end{array}$

Step 3

$\underline{\mathrm{A}=\mathrm{O}_{1}} \underline{\underline{\mathrm{O}_{7}}} \underline{\mathrm{B}=\mathrm{O}_{8}}: \underline{\mathrm{O}_{10}} \underline{\mathrm{B}-\mathrm{A}} \quad \underline{\text { Rank }}$

$\begin{array}{lllll}\text { Classroom } 3^{*} & 8.857 & 12.333 & 3.476 & 1\end{array}$

Sum of the ranks associated with the intervened classrooms $=1+1+1=3$; average $=(2.50+$ $2.50+3.476) / 3=2.825$

Note: * represents the classroom beginning the intervention on that particular step. |indicates the actual intervention start point for each classroom. 
Table 6. Design, Data, and Modified-Extended Revusky Partial Calculations for the Classroom Intervention Example

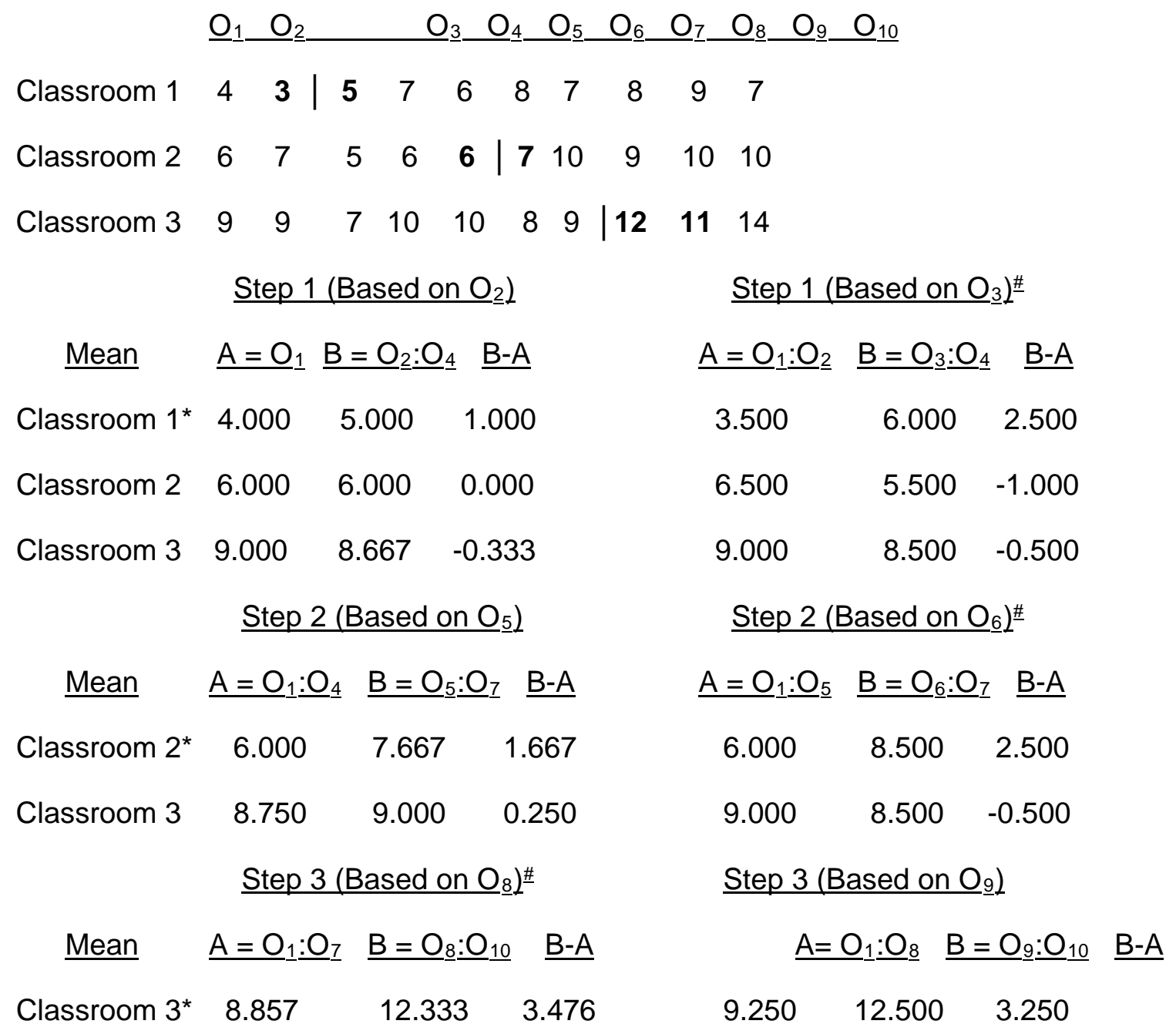

Note: The bolded values in the table represent the two-alternative intervention start-point interval that was specified for each classroom. | indicates the actual intervention start point for each classroom. * indicates the classroom that was actually intervened with on each step and \# indicates the actual intervention point for that classroom. 


\section{Figure Captions}

Figure 1. Hypothetical multiple-baseline design, depicting the performance outcomes (percent correct) of four students who received a sequentially introduced instructional intervention commencing immediately prior to Observations $6,9,12$, and 15, for Students 1-4 respectively.

Figure 2. Investigation 1: Comparison ( $\alpha=.05$, one-tailed) of the fixed intervention start-point Wampold-Worsham (WW), Revusky (Rev), and modified Revusky (Rev-M) randomization tests. The rejection rate of the null hypothesis is shown as a function of the effect size for a design with $N=4$ cases, 19 observations per case, an autocorrelation of 0 , a single intervention start point just prior to Observation 6 , and a stagger of: (A) one observation between intervention start points (i.e., start points of 6 , 7, 8, 9, (B) two observations between intervention start points (i.e., start points of 6, 8, 10, 12), and (C) three observations between intervention start points (i.e., start points of $6,9,12,15$.

Figure 3. Investigation 1: Comparison ( $\alpha=.05$, one-tailed) of the fixed intervention start-point Wampold-Worsham (WW), Revusky (Rev), and modified Revusky (Rev-M) randomization tests. The rejection rate of the null hypothesis is shown as a function of the effect size for a design with $N=4$ cases, 19 observations per case, an autocorrelation of .30 , a single intervention start point just prior to Observation 6 , and a stagger of: $(A)$ one observation between the successive cases' intervention start points (i.e., start points of $6,7,8,9)$, (B) two observations between intervention start points (i.e., start points of $6,8,10,12$ ), and (C) three observations between intervention start points (i.e., start points of $6,9,12,15$ ).

Figure 4. Investigation 1: Comparison ( $\alpha=.05$, one-tailed) of the fixed intervention start-point Wampold-Worsham (WW), Revusky (Rev), and modified Revusky (Rev-M) 
randomization tests. The rejection rate of the null hypothesis is shown as a function of the effect size for designs having $N=5$ cases (WW5, Rev5, Rev-M5) with 22 observations per case and for $N=6$ cases (WW6, Rev6, Rev-M6) with 25 observations per case, an autocorrelation of 0 , a single intervention start point just prior to Observation 6, and a stagger of: $(A)$ one observation between intervention start points (e.g., start points of $6,7,8,9,10)$, (B) two observations between intervention start points (e.g., start points of $6,8,10,12,14)$, and (C) three observations between intervention start points (e.g., start points of $6,9,12,15,18)$.

Figure 5. Investigation 1: Comparison ( $\alpha=.05$, one-tailed) of the fixed intervention start-point Wampold-Worsham (WW), Revusky (Rev), and modified Revusky (Rev-M) randomization tests. The rejection rate of the null hypothesis is shown as a function of the effect size for designs having $N=5$ cases (WW5, Rev5, Rev-M5) with 22 observations per case and for $N=6$ cases (WW6, Rev6, Rev-M6) with 25 observations per case, an autocorrelation of .30, a single intervention start point just prior to Observation 6, and a stagger of: $(A)$ one observation between intervention start points (e.g., start points of 6, 7, 8, 9, 10), (B) two observations between intervention start points (e.g., start points of $6,8,10,12,14)$, and (C) three observations between intervention start points (e.g., start points of $6,9,12,15,18)$.

Figure 6. Investigation 2: Comparison ( $\alpha=.05$, one-tailed) of the random intervention-start-point Koehler-Levin procedure with two $[\mathrm{KL}(2)]$ or three $[\mathrm{KL}(3)]$ potential intervention start points per case, the modified Revusky procedure with two [Rev-M(2)] or three [Rev$M(3)]$ potential intervention start points, and the restricted Marascuilo-Busk procedure with a between-case stagger of at least one observation (MB-R/1), when the autocorrelation is .30 . The rejection rate of the null hypothesis is shown as a function of the effect size for designs that have: (A) $N=4$ cases with 19 observations per case, where the first potential intervention start points for the four cases are $6,9,12$, and 15 
for the $\mathrm{KL}(2), \mathrm{KL}(3)$, Rev-M(2), and Rev-M(3) designs, whereas the $M B-R / 1$ design had start points selected at random without replacement from the interval 6 to 15 inclusive; and (B) $N=5$ cases with 22 observations per case, where the first potential intervention start points for the five cases are 6, 912,15 , and 18 for $K L(2), K L(3)$, Rev$M(2)$, and Rev-M(3) designs, whereas the MB-R/1 design had start points selected at random without replacement from the interval 6 to 18 inclusive.

Figure 7. Investigation 3: Comparison ( $\alpha=.05$, one-tailed) of the Wampold-Worsham procedure with a three-point stagger (WW/3), the Koehler-Levin procedure with either two [(KL(2)] or three $[(\mathrm{KL}(3)]$ potential intervention start points per case, and the restricted Marascuilo-Busk procedure with a between-case stagger of at least either one (MB$\mathrm{R} / 1$ ) or two (MB-R/2) observations. The rejection rate of the null hypothesis is shown as a function of the effect size for an autocorrelation of .30 for designs with: (A) $N=3$ cases with 16 observations per case, (B) $N=4$ cases with 19 observations per case, and (C) $N=5$ cases with 22 observations per case.

Figure 8. Investigation 4: Comparison ( $\alpha=.05$, one-tailed) of the Wampold-Worsham procedure with a between-case stagger of three observations (WW/3), the Koehler-Levin procedure with either two $[\mathrm{KL}(2)]$ or three $[\mathrm{KL}(3)]$ potential intervention start points per case, the restricted Marascuilo-Busk procedure with a between-case stagger of at least one observation (MB-R/1), the modified Revusky procedure with one potential intervention start point per case [Rev-M(1)], which is equivalent in power to the original Revusky procedure in the $N=4$ design (see Investigation 1 ), and the modified Revusky procedure with either two [Rev-M(2)] or three [Rev-M(3)] potential intervention start points per case. The rejection rate of the null hypothesis is shown as a function of the effect size for designs having $N=4$ cases with 19 observations per case, an autocorrelation of .30, and effects that were: (A) delayed by one observation 
and abrupt, (B) delayed by two observations and abrupt, and (C) immediate and gradual.

Figure 9. Investigation 4: Comparison ( $\alpha=.05$, one-tailed) of the Wampold-Worsham procedure with a between-case stagger of three observations (WW/3), the Koehler-Levin procedure with either two $[\mathrm{KL}(2)]$ or three $[\mathrm{KL}(3)]$ potential intervention start points per case, the restricted Marascuilo-Busk with a between-case stagger of at least one observation (MB-R/1), the modified Revusky procedure with either one [Rev-M(1)], two [Rev-M(2)], or three [Rev-M(3)] potential intervention start points per case, and the original Revusky procedure with a between-case stagger of three observations (Rev/3). The rejection rate of the null hypothesis is shown as a function of the effect size for designs having $N=5$ cases with 22 observations per case, an autocorrelation of .30, and effects that were: (A) delayed by one observation and abrupt, $(B)$ delayed by two observations and abrupt, and (C) immediate and gradual. 


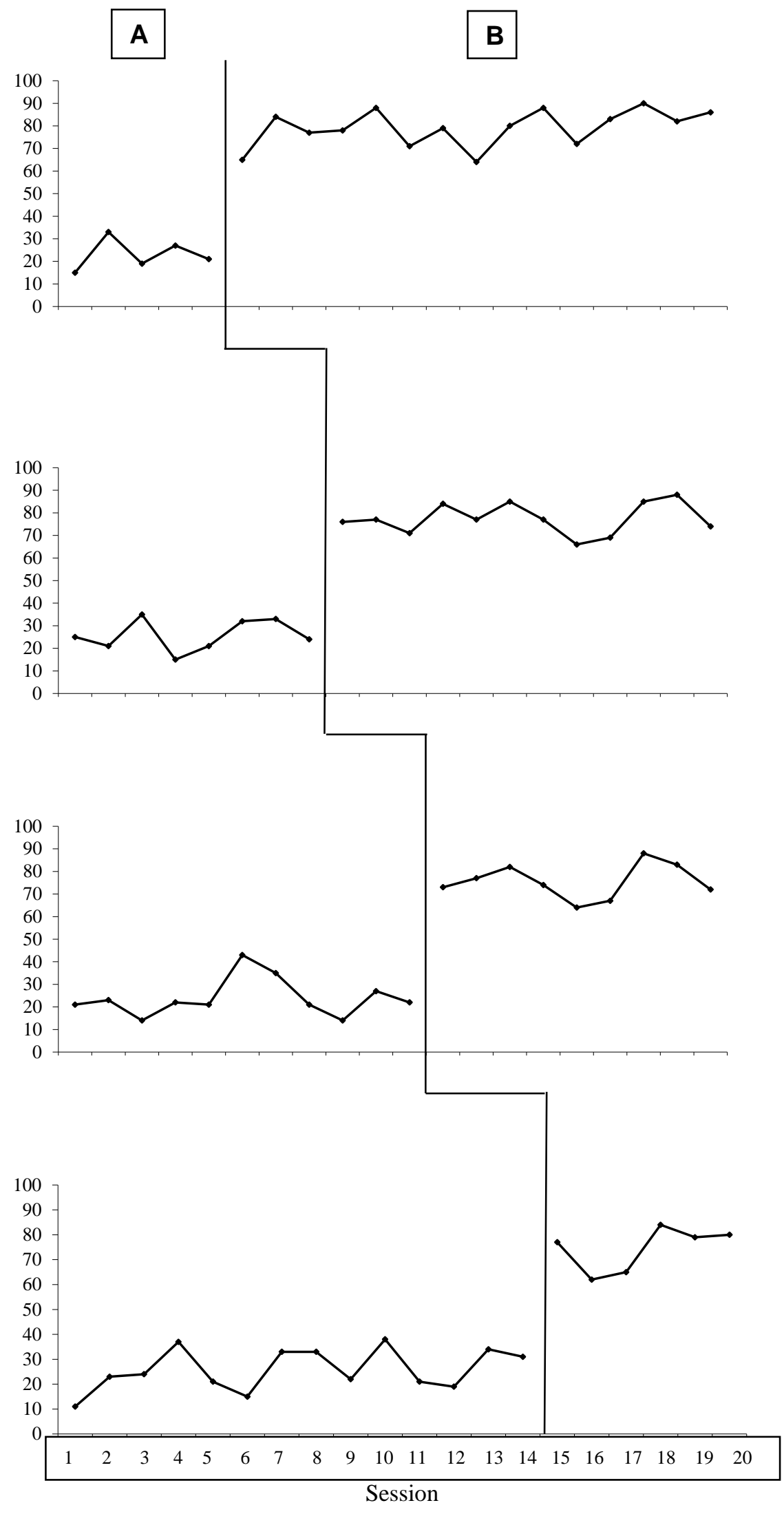



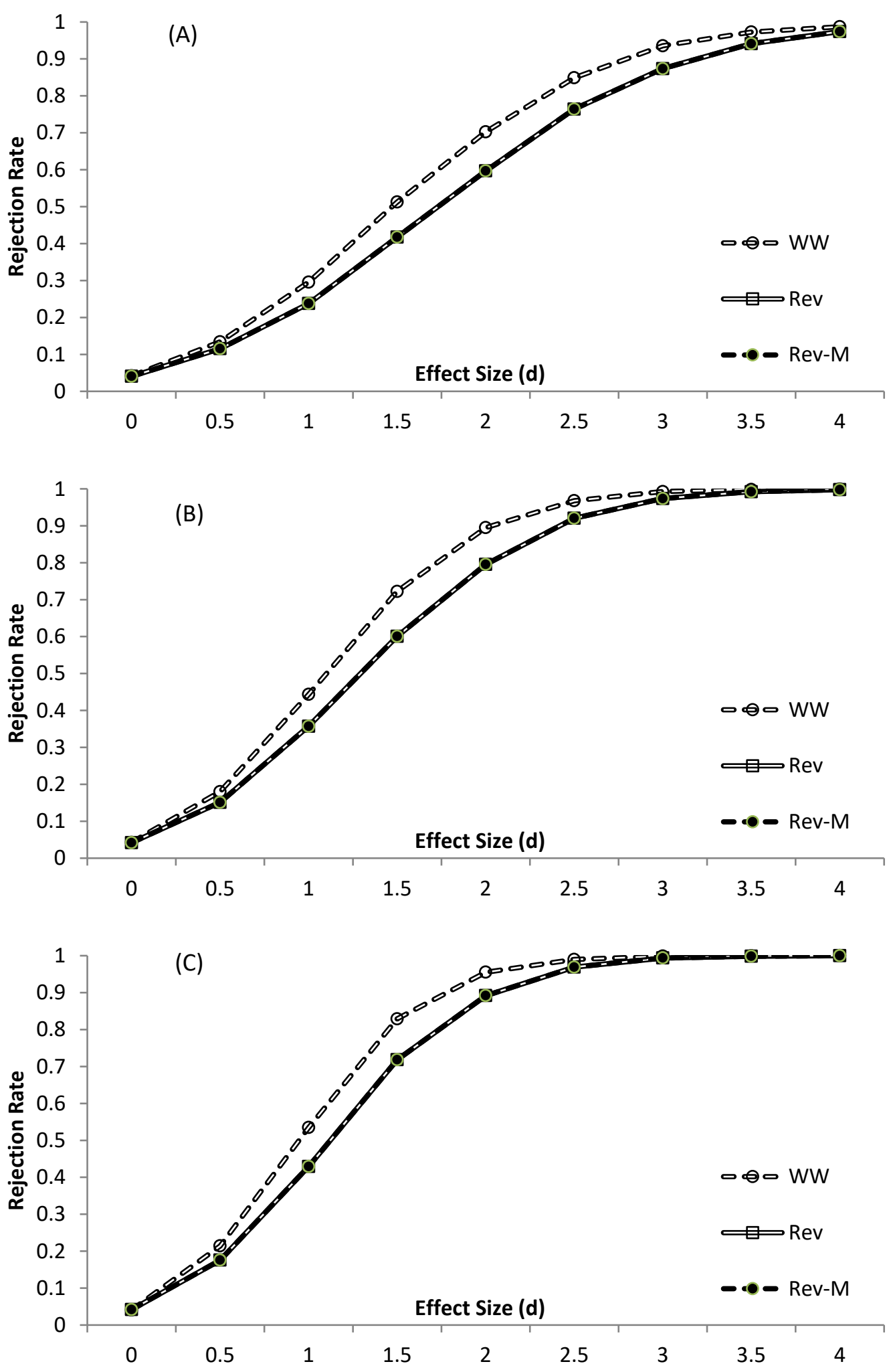

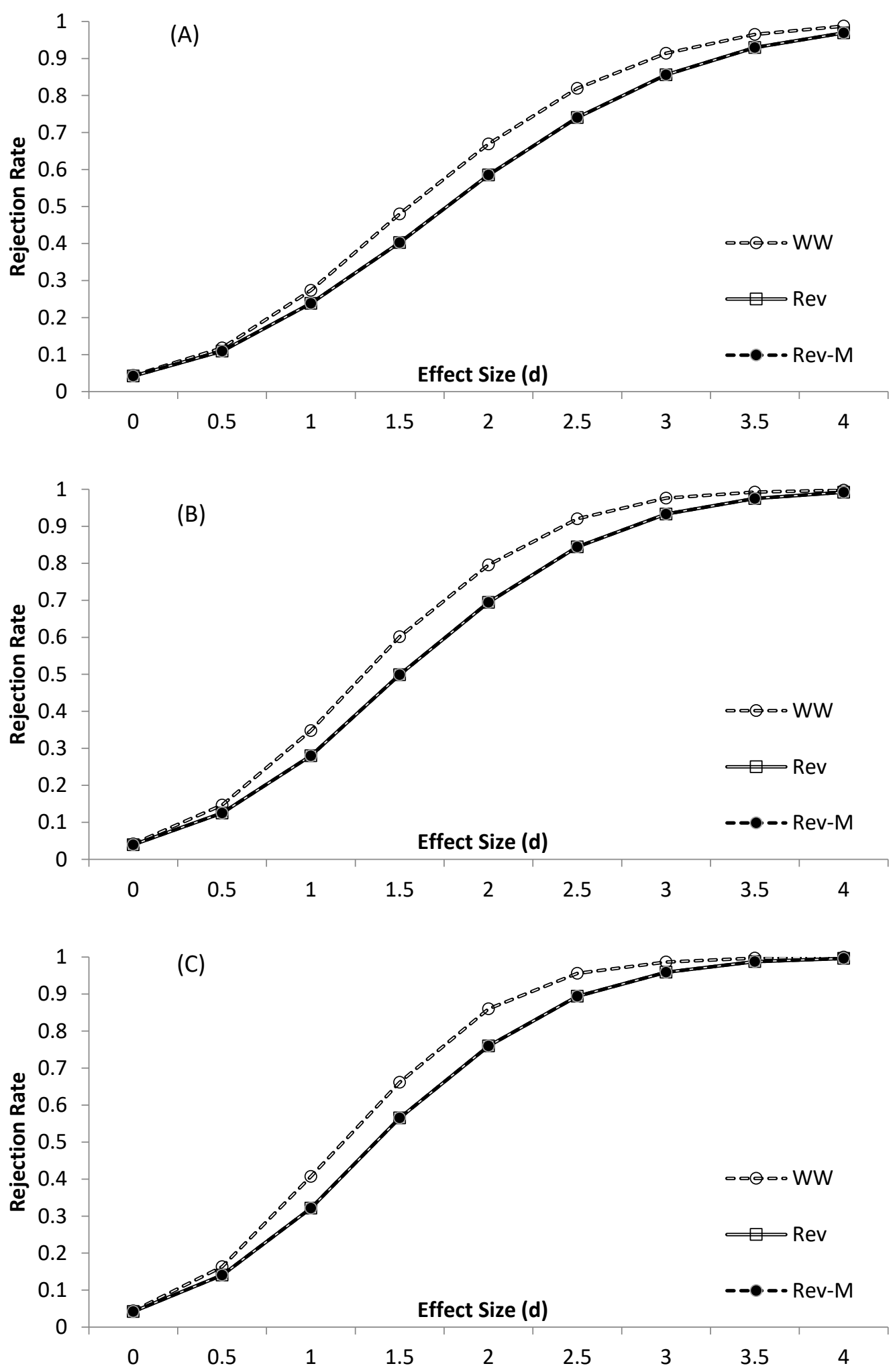

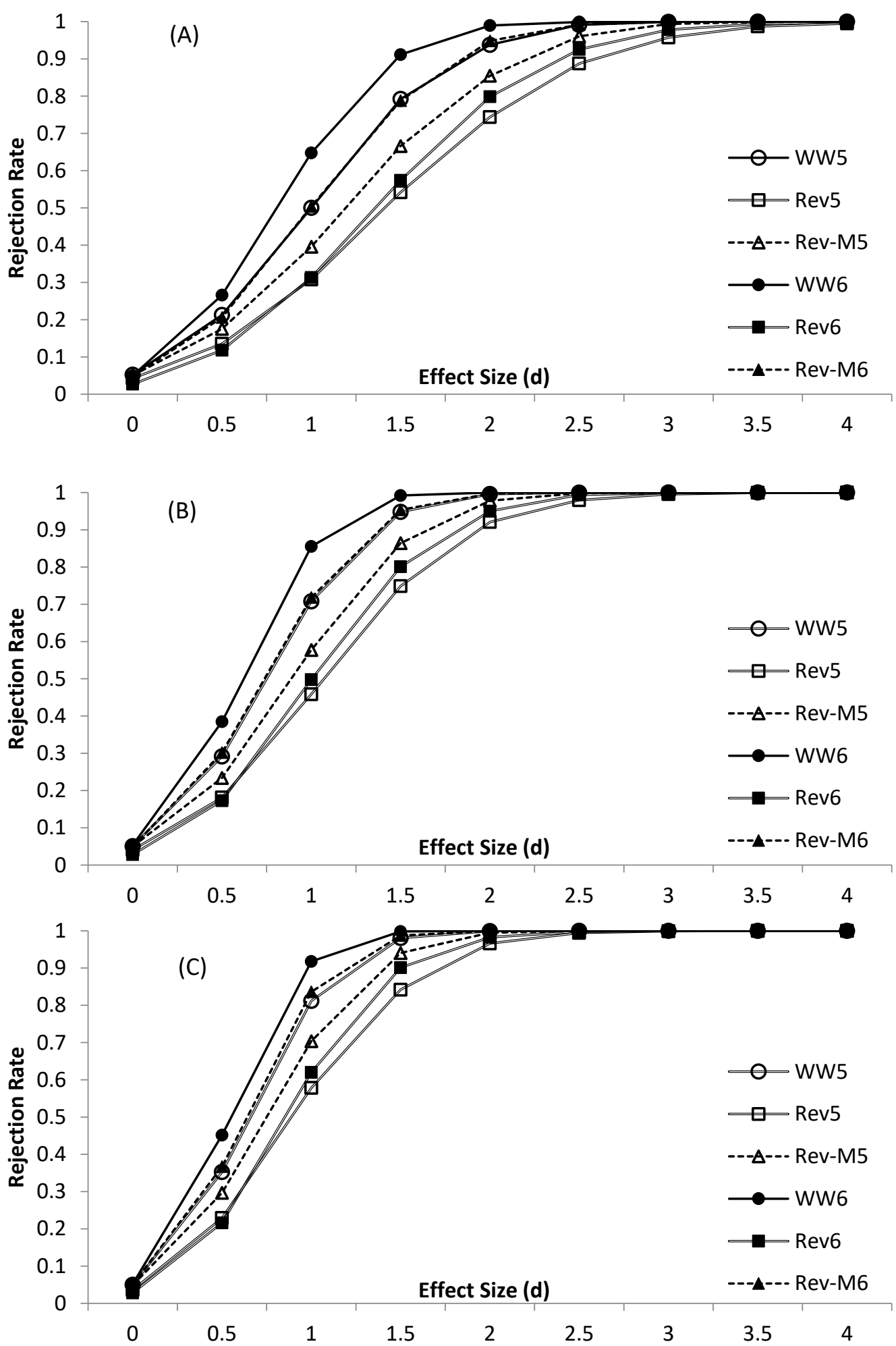

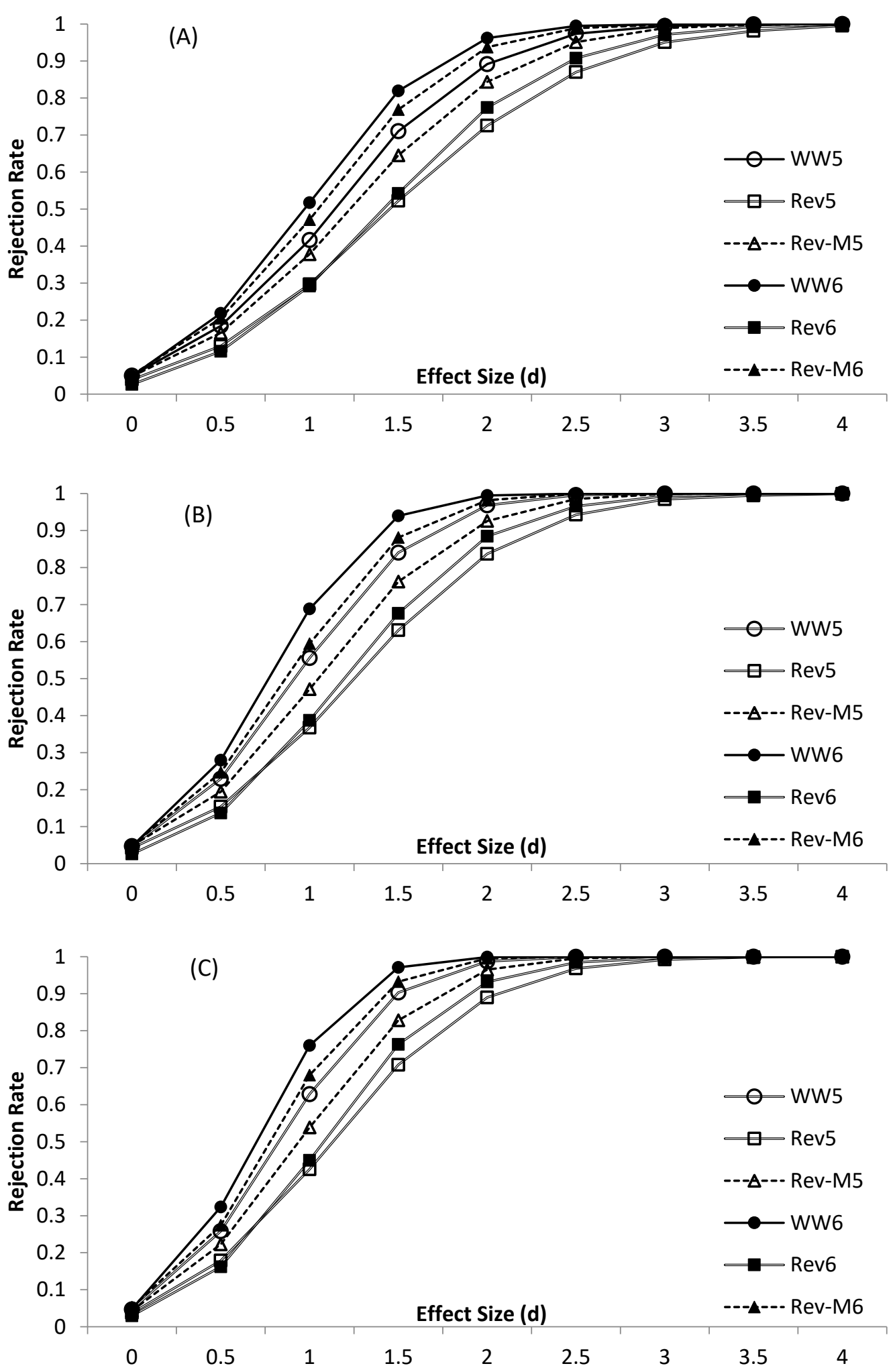

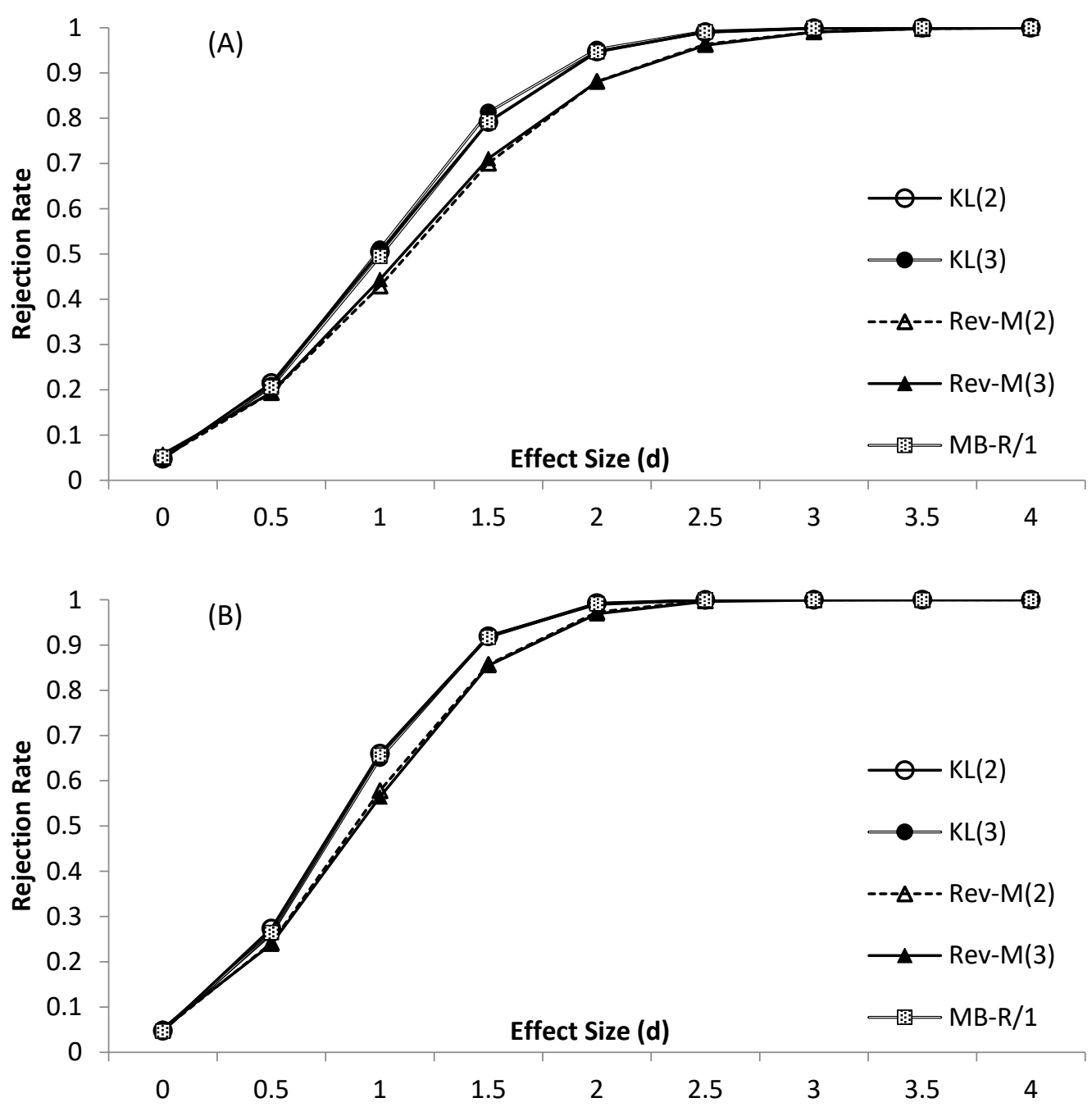

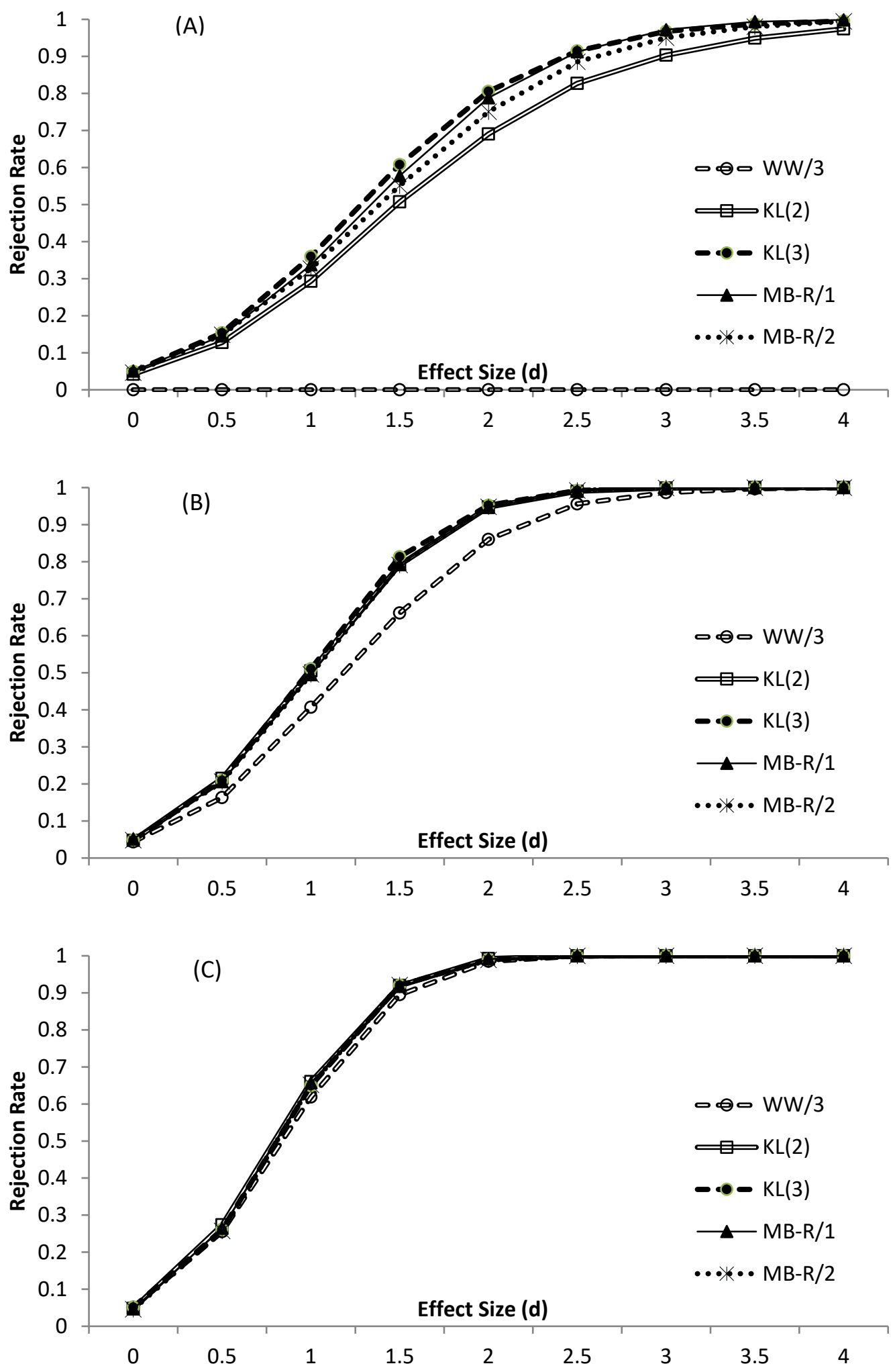

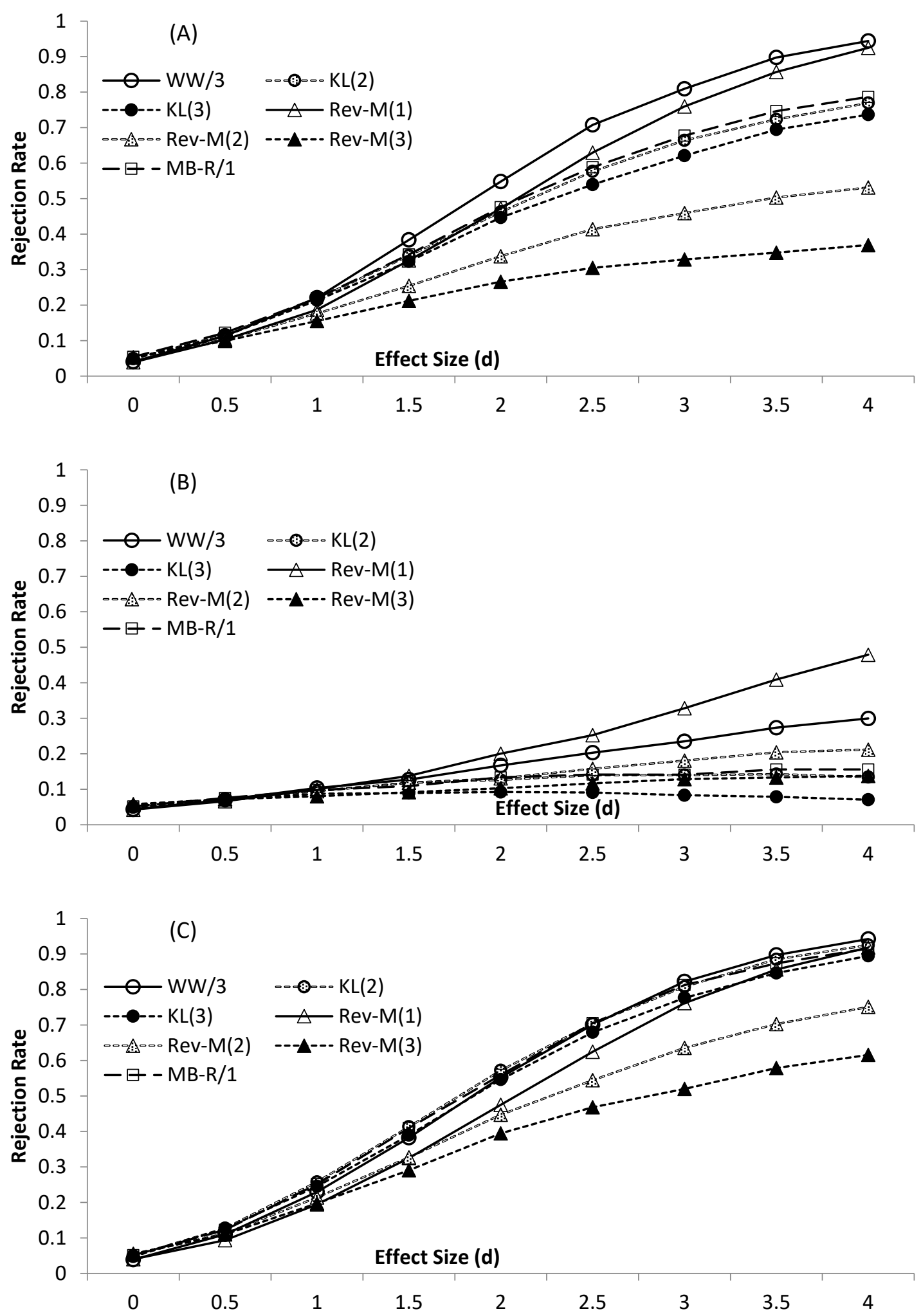

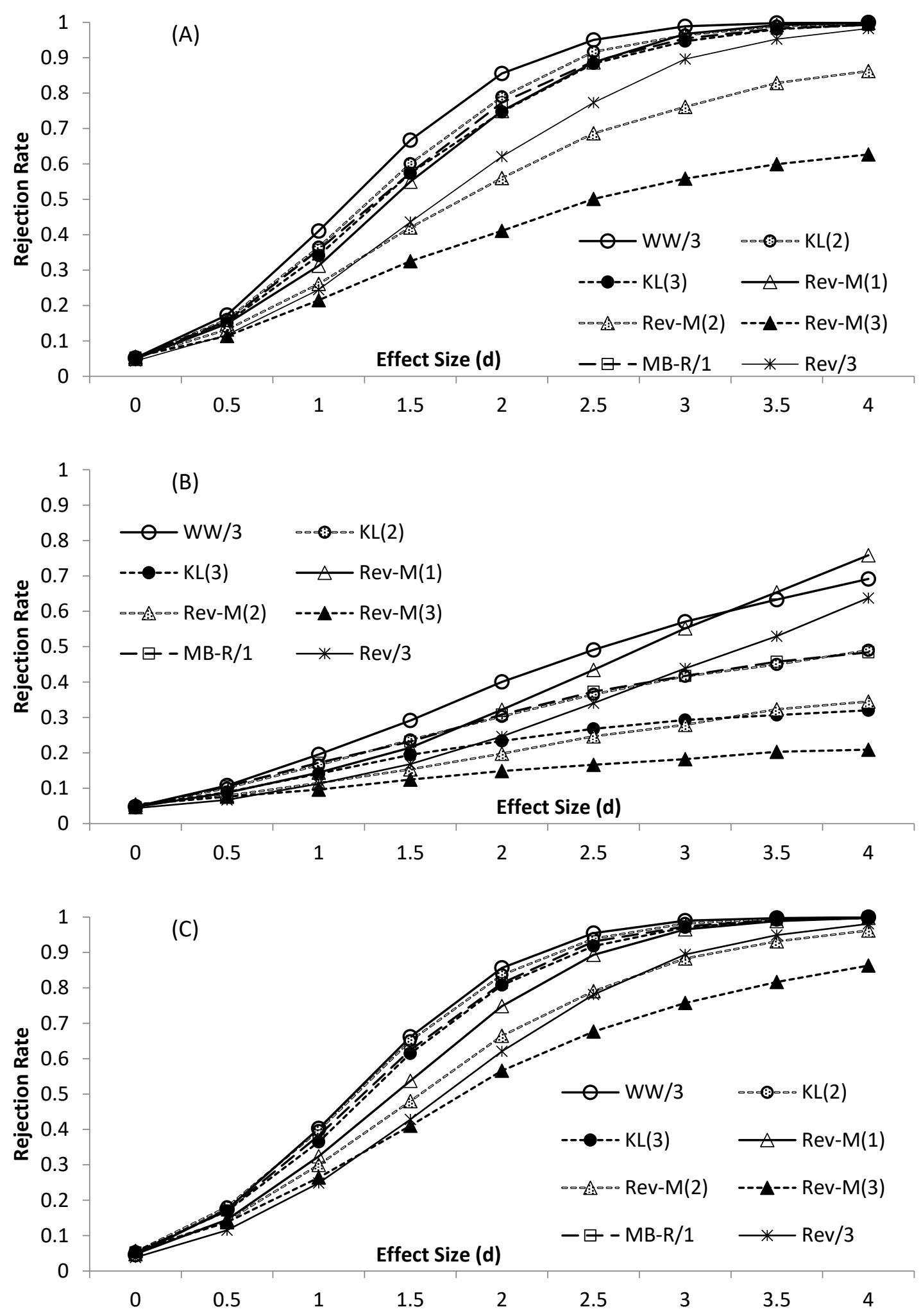